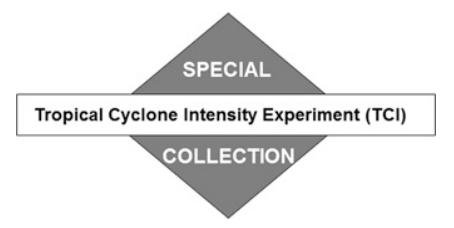

\title{
Axisymmetric Potential Vorticity Evolution of Hurricane Patricia (2015)
}

\author{
JonAthan MARTinez AND Michael M. BELL \\ Department of Atmospheric Science, Colorado State University, Fort Collins, Colorado \\ ROBERT F. ROGERS \\ Hurricane Research Division, NOAA/Atlantic Oceanographic and Meteorological Laboratory, Miami, Florida \\ JAMES D. DOYLE \\ U.S. Naval Research Laboratory, Monterey, California
}

(Manuscript received 21 December 2018, in final form 19 April 2019)

\begin{abstract}
Operational numerical models failed to predict the record-setting rapid intensification and rapid overwater weakening of Hurricane Patricia (2015) in the eastern North Pacific basin, resulting in large intensity forecast errors. In an effort to better understand the mesoscale processes contributing to Patricia's rapid intensity changes, we analyze high-resolution aircraft observations collected on 22-23 October. Spline-based variational analyses are created from observations collected via in situ measurements, Doppler radar, and fulltropospheric dropsonde profiles as part of the Office of Naval Research Tropical Cyclone Intensity (TCI) experiment and the National Oceanic and Atmospheric Administration Intensity Forecasting Experiment (IFEX). We present the first full-tropospheric calculation of the dry, axisymmetric Ertel's potential vorticity (PV) in a tropical cyclone without relying on balance assumptions. Detailed analyses reveal the formation of a "hollow tower" PV structure as Patricia rapidly approached its maximum intensity, and a subsequent breakdown of this structure during Patricia's rapid overwater weakening phase. Transforming the axisymmetric PV analyses from radius-height to potential radius-isentropic coordinates reveals that Patricia's rapid intensification was closely related to the distribution of diabatic heating and eddy mixing. During Patricia's rapid overwater weakening phase, eddy mixing processes are hypothesized to be the primary factor rearranging the PV distribution near the eye-eyewall region, diluting the PV previously confined to the hollow tower while approximately conserving the absolute circulation.
\end{abstract}

\section{Introduction}

Accurate forecasts of tropical cyclone (TC) intensity changes remain one of the most difficult weather predictions, even for short lead times. This is in part due to multiscale interactions, which require operational forecast models to precisely capture the evolution of the atmosphere over a vast range of scales in the vicinity of a TC. DeMaria et al. (2014) demonstrated that although intensity forecast errors have not improved as much as track forecast errors over the past few decades, intensity forecasts at long lead times (e.g., 48-120h) have improved at a statistically significant rate. However, only marginal improvements have been made at shorter lead

\footnotetext{
Corresponding author: Jonathan Martinez, jon.martinez@ colostate.edu
}

times (e.g., 24-48 h), suggesting that additional research is required to better understand the underlying mechanisms associated with TC intensification. In the case of eastern North Pacific Hurricane Patricia (2015), extreme rapid intensification was not well predicted by either global or mesoscale models (Rogers et al. 2017, their Fig. 1), which contributed to a 48-h intensity forecast error of $105 \mathrm{kt}\left(1 \mathrm{kt} \approx 0.51 \mathrm{~m} \mathrm{~s}^{-1}\right.$; Kimberlain et al. 2016). In this study, we utilize high-resolution observations collected during the Office of Naval Research (ONR) Tropical Cyclone Intensity experiment (TCI; Doyle et al. 2017) and the National Oceanic and Atmospheric Administration (NOAA) Intensity Forecasting Experiment (IFEX; Rogers et al. 2006, 2013b) to examine Hurricane Patricia's structural evolution during its record-setting rapid intensification and overwater weakening. Combining new observational capabilities 
from high-density dropsondes and airborne Doppler radar data, we calculate the full-tropospheric, dry axisymmetric Ertel's potential vorticity (PV; Ertel 1942) within the inner-core region of Patricia without relying on balance assumptions. We utilize these unprecedented observations of $\mathrm{PV}$ to elucidate the mesoscale processes contributing Patricia's rapid intensity changes, and to better understand the evolution of the strongest TC observed to date in the Western Hemisphere (Rogers et al. 2017).

The current state of forecasting TC rapid intensification (RI) events is largely dependent on probabilistic techniques, which aid the intensity guidance provided by deterministic models. For example, the rapid intensification index (RII; Kaplan et al. 2010) employs large-scale environmental predictors from the Statistical Hurricane Intensity Prediction Scheme (SHIPS; DeMaria et al. 2005) to estimate the probability of RI. The skill of the SHIPS-RII has shown improvements when considering inner-core predictors in addition to these large-scale environmental predictors (Kaplan et al. 2015; Rozoff et al. 2015). Furthermore, given a favorable preexisting environment, inner-core processes have been suggested to be the primary influence on TC intensification rates (Hendricks et al. 2010). Therefore, improving our understanding of the internal processes within a TC can lead to more skillful intensity forecasts.

A common theoretical and numerical framework employed to understand TC intensification is predicated on the balanced vortex model (Eliassen 1951), whereby an axisymmetric vortex is assumed to continuously evolve in a state of gradient wind and hydrostatic balance in response to a specified (often time invariant) forcing. Within the balanced vortex framework, it has been shown that heating concentrated radially inward of the radius of maximum tangential winds (RMW) is more favorable for TC intensification (Shapiro and Willoughby 1982; Pendergrass and Willoughby 2009), which has been argued to be due to increased heating efficiency (Schubert and Hack 1982; Hack and Schubert 1986; Pendergrass and Willoughby 2009; Vigh and Schubert 2009) and the convergence of lower-tropospheric angular momentum surfaces toward the heat source via a transverse circulation response (Smith and Montgomery 2016; Smith et al. 2018). While frictional forcing also plays a role, in this framework the evolution of a TC is primarily governed by sources of heating in the eyewall, which drive the simultaneous development of a primary and secondary circulation. Central to this framework is the inextricable evolution of the wind and mass fields, both inherent to the quantity known as Ertel's PV. The "invertibility" principle (Hoskins et al. 1985) states that through specification of a balanced state for the flow of interest (e.g., gradient wind and hydrostatic balance in a TC) and suitable boundary conditions, Ertel's $\mathrm{PV}$ can be inverted to recover the balanced mass and wind fields (Schubert and Hack 1983; Schubert and Alworth 1987). It can be shown in the steady state, above the frictional boundary layer, that the axisymmetric diabatic heating and PV become locked with one another (Hausman et al. 2006). The emergent structure during TC intensification within this framework is characterized by a "hollow tower" of PV where the maximum PV is radially displaced from the axis of rotation and concentrated along the inner edge of the RMW (Möller and Smith 1994).

Prior studies have demonstrated that the radial PV gradients in a TC serve as waveguides for vortex Rossby waves (VRWs; Guinn and Schubert 1993; Montgomery and Kallenbach 1997). In the presence of a hollow-tower PV structure, the sign reversal of the radial PV gradient satisfies the Rayleigh necessary condition for barotropic instability (Schubert et al. 1999), or more generally, the Charney-Stern necessary condition for combined barotropic-baroclinic instability (Montgomery and Shapiro 1995). Exponential instability resulting from phase locking and mutual amplification of counterpropagating VRWs can lead to a breakdown of the PV ring and mixing at the eye-eyewall interface (Schubert et al. 1999; Kossin and Schubert 2001; Hendricks et al. 2009; Hendricks and Schubert 2010). Asymmetric PV structures emerging from this instability can act as a "transient intensification break" during symmetric intensification, although consecutive PV mixing events can result in the vortex achieving a higher intensity than it would have in its absence because of the cumulative transport of high PV air into the eye region (Rozoff et al. 2009). Furthermore, the PV ring can roll up into convective mesovortices, which simultaneously decrease the point-minimum pressure and increase the point-maximum winds of the vortex (Hendricks et al. 2012, 2014). Möller and Montgomery $(1999,2000)$ proposed a "convective pulsing" intensification mechanism whereby inner-core PV asymmetries induced by repetitive convective bursts in the eyewall are axisymmetrized, spinning up the symmetric vortex. Collectively, the aforementioned studies depict a conceptual model for understanding TC intensification where the evolution of PV in the presence of diabatic heating is a leading-order process.

Calculation of PV in a TC from observations is difficult, as it requires dense sampling to calculate both kinematic and thermodynamic gradients. Because of these limitations, observational studies have typically used vertical vorticity as a proxy for PV. Doppler radar data and in situ measurements have been utilized in both composite and case studies to demonstrate that intensifying TCs are characterized by a ringlike structure of 
vorticity with a maximum located off the axis of rotation, radially inward of the RMW (Kossin and Eastin 2001; Rogers et al. 2013a, 2015; Martinez et al. 2017). Kossin and Eastin (2001) also demonstrated that intensifying TCs possess relatively lower angular velocity and equivalent potential temperature in the eye compared to the eyewall. The composite study of Rogers et al. (2013a) further noted that convective bursts (CBs) are preferentially located radially inward of the RMW for intensifying TCs compared to radially outward of the RMW for steady-state TCs; the former structure was observed in detail during the rapid intensification of Hurricane Earl (2010; Rogers et al. 2015). Martinez et al. (2017) noted that once a TC reaches major hurricane intensity $\left(50 \mathrm{~m} \mathrm{~s}^{-1}\right)$, a ring of vorticity can be associated with further intensification or subsequent weakening, suggesting that additional physical processes may become important near peak intensity of the strongest TCs.

The purpose of this study is to improve our understanding of the rapid intensification and weakening of Hurricane Patricia (2015) using the first full-tropospheric, axisymmetric observational analysis of PV in a TC without relying on balance assumptions. High-resolution observations collected during both ONR TCI and NOAA IFEX are utilized to construct spline-based variational analyses. The field observations provide an opportunity to examine the full-tropospheric PV structure and evolution of Hurricane Patricia during its rapid intensification and weakening phases. A PV framework further provides a natural dynamical perspective to better understand the inner-core structural evolution of a TC experiencing rapid intensity changes. Section 2 introduces Patricia's evolution along with the intensive observing periods during which Patricia was sampled. Section 3 describes the multiple observing platforms, quality control processes for the observations, and the variational analysis methodology. Section 4 presents the axisymmetric inner-core PV structure and evolution of Hurricane Patricia during its rapid intensification and weakening phases. A discussion of the physical mechanisms contributing to Patricia's rapid intensity changes will be provided in section 5 , and finally, conclusions and future work will be presented in section 6 .

\section{Synopsis of Hurricane Patricia}

Patricia was first declared a tropical depression at 0600 UTC 20 October approximately 180 nautical miles $(\sim 330 \mathrm{~km})$ south-southeast of Salina Cruz, Mexico (Fig. 1). After being declared a tropical storm 18-h after genesis, Patricia drifted west-southwest through a slightly unfavorable thermodynamic environment prior to entering
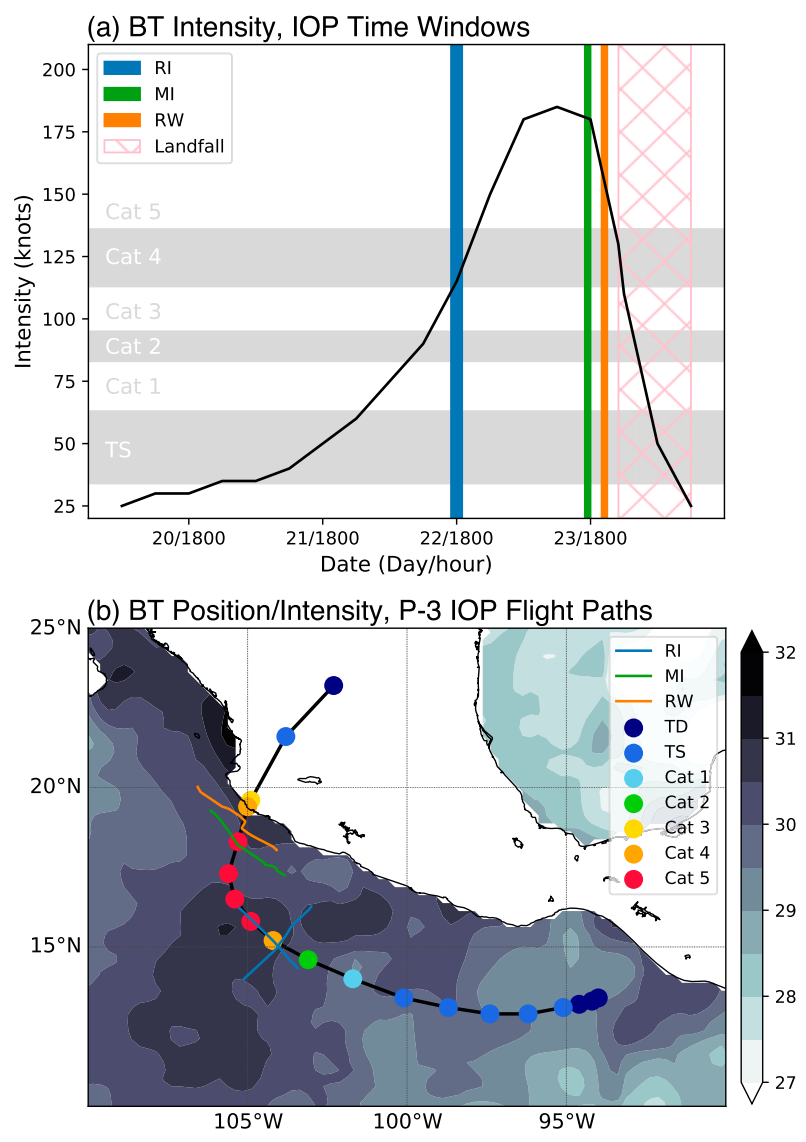

FIG. 1. (a) National Hurricane Center (NHC) Best Track (BT) intensity (kt) for Hurricane Patricia (2015). Colored lines denote the time windows for each intensive observing period (IOP) as follows: rapid intensification (RI): 1715-1915 UTC 22 Oct; near maximum intensity (MI): 1710-1800 UTC 23 Oct; and rapid weakening (RW): 2015-2100 UTC 23 Oct. (b) BT position (black curve) and SaffirSimpson scale intensity (colored dots) throughout Patricia's life cycle are overlaid on the sea surface temperatures (shaded, K) valid 19 Oct from the Optimum Interpolation Sea Surface Temperature database (Banzon et al. 2016). The colored lines denote the P-3 flight paths corresponding to the IOPs.

an environment characterized by high oceanic heat content and sea surface temperatures (Fig. 1b), conducive for rapid organization and development (Kimberlain et al. 2016). Shortly after this time, Patricia rapidly intensified $54 \mathrm{~m} \mathrm{~s}^{-1}$ in $24 \mathrm{~h}$, transitioning from a 75-kt category 1 hurricane at 0600 UTC 22 October to a 180-kt category 5 major hurricane. Six hours later, Patricia attained its maximum lifetime intensity of $185 \mathrm{kt}$ with a minimum central pressure of $872 \mathrm{hPa}$. Both the extreme rapid intensification and maximum lifetime intensity (defined as the maximum 1-min sustained wind speed $10 \mathrm{~m}$ above the ocean) of Patricia were unprecedented in global observations, placing it in the "tropical record books" [see Table 2 of Rogers et al. (2017)]. This dramatic intensification was not well predicted by either global or 
TABLE 1. The name of each IOP for Hurricane Patricia (2015) analyzed in this study and its corresponding time window.

\begin{tabular}{lc}
\hline \hline \multicolumn{1}{c}{ IOP } & Analysis time \\
\hline Rapid intensification (RI) & $1715-1915$ UTC 22 Oct \\
Near maximum intensity (MI) & $1710-1800$ UTC 23 Oct \\
Rapid weakening (RW) & $2015-2100$ UTC 23 Oct \\
\hline
\end{tabular}

mesoscale models [see Fig. 1 of Rogers et al. (2017)], which resulted in a 48-h operational intensity forecast error of $105 \mathrm{kt}$ (Kimberlain et al. 2016). Equally noteworthy was Patricia's rapid overwater weakening and filling of $\sim 26 \mathrm{~m} \mathrm{~s}^{-1}$ and $54 \mathrm{hPa}$ in $5 \mathrm{~h}$, respectively, prior to making landfall as a category 4 major hurricane in the Mexican state of Jalisco.

High-resolution observations gathered in Patricia's inner core during both TCI and IFEX provide the unique opportunity to explore the mechanisms contributing to such remarkable intensity changes. Coordinated flight missions between the National Aeronautics and Space Administration (NASA) WB-57 and the NOAA WP-3D (P-3) aircraft were executed from 21 to 23 October. For the sake of organization, we define an intensive observing period (IOP) for each time period that observations were collected in Patricia by both the P-3 and WB-57. The time window for each IOP is defined with respect to the time Patricia was sampled by the P-3 tail Doppler radar. Table 1 provides a brief summary of the three IOPs analyzed in this study and their corresponding time windows. An earlier IOP took place on 21 October 2015 as Patricia began the early stages of its intensification, and was not analyzed in this study. Future work will investigate the structure of Patricia during this earlier stage of organization and rapid development.

Observations collected between 1715 and 1915 UTC 22 October as Patricia was in the midst of its recordbreaking rapid intensification phase (Fig. 1a) are denoted herein as the RI IOP. Microwave imagery shows deep convection in the eyewall wrapping around a compact center of circulation as Patricia had just achieved category 4 status (Fig. 2a). Both the P-3 and WB-57 executed figure-4 patterns with the P-3 flying the 700-hPa flight level (blue path shown in Fig. 1b) and the WB-57 flying at $18-\mathrm{km}$ altitude.

On 23 October, as the P-3 traversed Patricia's inner core at the $700-\mathrm{hPa}$ flight level, it encountered severe turbulence, forcing the crew to loiter and recover on the southeastern side of Patricia for $\sim 2 \mathrm{~h}$ before completing the second center pass (Rogers et al. 2017). Therefore, observations are divided into a "near maximum intensity" (MI) IOP from 1710 to 1800 UTC, and a "rapid weakening" (RW) IOP from 2015 to 2100 UTC. The P-3 flight tracks for these two IOPs are shown by the green and orange paths, respectively, in Fig. 1b. A single WB-57 overpass at $18-\mathrm{km}$ altitude coincided with the RW IOP (second P-3 center pass on 23 October). Microwave imagery approximately $4 \mathrm{~h}$ before the first P-3 center pass highlights Patricia's remarkably compact eyewall and corresponding deep convection along with a pronounced principal rainband (Fig. 2b). A dramatic structural change is observed $\sim 5 \mathrm{~h}$ later in the microwave imagery as Patricia approached the coast of Mexico, showing the development of a concentric eyewall structure (Fig. 2c). A detailed description of each observing platform is given in the following section along with the method of creating the observational analyses for each IOP.

\section{Datasets and analysis methodology}

The WB-57 was equipped with the High Definition Sounding System (HDSS) designed to simultaneously receive data from multiple dropsondes. As the WB-57 flew above the inner core of Hurricane Patricia at approximately 18-km altitude, Expendable Digital Dropsondes (XDDs) were released in a "rapid fire" sequence resulting in a spatial interval of $\sim 4 \mathrm{~km}$ between each XDD. The XDDs are global positioning system (GPS) dropsondes designed without a parachute, containing grooves etched into the polystyrene plastic-printed circuit board to provide stability during rapid descent (Black et al. 2017). Pressure, temperature, and relative humidity (PTH) are recorded along the XDD trajectories with a 2-Hz sampling frequency, and horizontal velocity measurements are recorded with a 4-Hz sampling frequency. The HDSS has been successfully tested and evaluated on multiple platforms and in different environments prior to its implementation during TCI, showing robust results and strong agreement with measurements made by Vaisala RD94 GPS dropsondes (Black et al. 2017). Pressure, temperature, relative humidity, and horizontal velocity errors are reported as $1.5 \mathrm{hPa}, 0.14^{\circ} \mathrm{C}, 1.8 \%$, and $0.1 \mathrm{~m} \mathrm{~s}^{-1}$, respectively (Bell et al. 2016; Black et al. 2017). Each sounding is subjected to a combined objective-subjective quality control procedure, described in detail by Bell et al. (2016), to ensure reliable data quality for the final product.

The P-3 tail Doppler radar is an X-band $(\sim 3-\mathrm{cm}$ wavelength) radar with two antennas, one scanning fore and the other scanning aft, both positioned at an $\sim 20^{\circ}$ offset from the aircraft's longitudinal axis. This separation allows for a dual-Doppler synthesis of the observed Doppler velocities from both the fore and aft scanning radars. The radar data are corrected for navigational errors using the method described by Cai et al. (2018) and subject to an automated quality control process following the "medium threshold" algorithm 

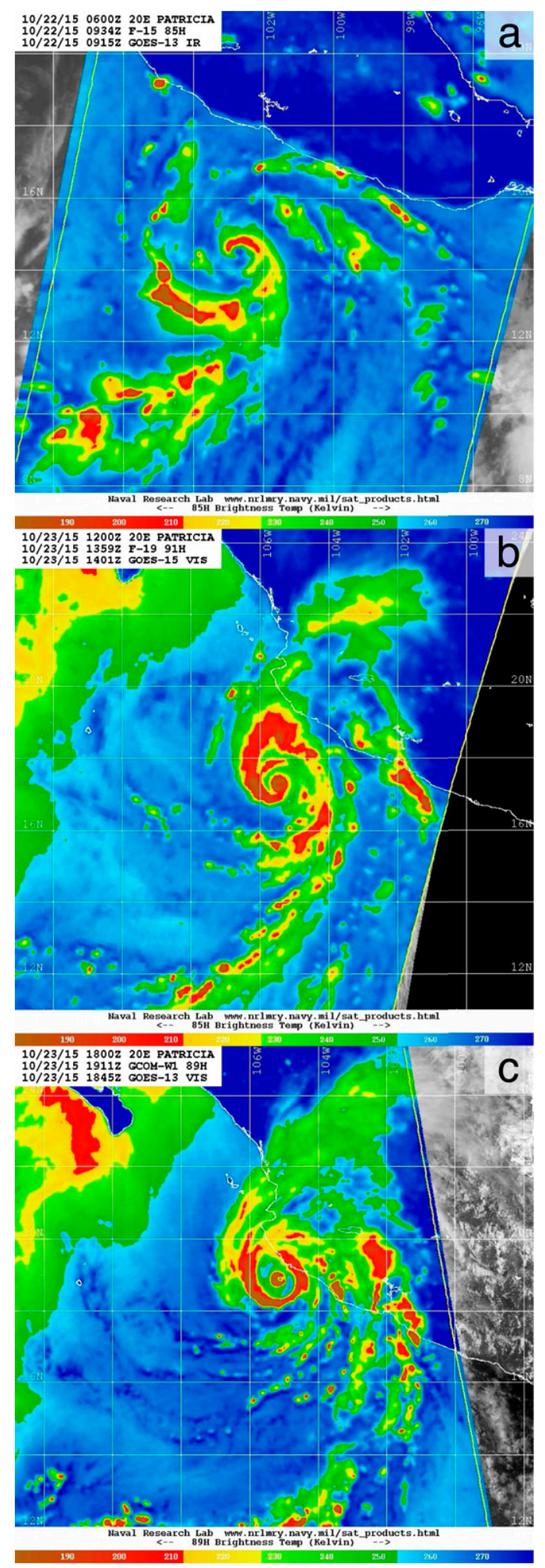

FIG. 2. Horizontal-polarized microwave $(85-91 \mathrm{GHz})$ brightness temperature structure of Hurricane Patricia from 22 to 23 Oct as observed by the Defense Meteorological Satellite Program (DMSP) and Global Change Observation Mission-Water "Shizuku" $(G C O M-W)$ polar-orbiting satellites. The microwave imagery is described by Bell et al. (2013). Additional quality control of the radar observations is carried out manually to remove ocean returns, radar sidelobes, and secondtrip echoes.

The HDSS and tail Doppler radar observations constitute the majority of the data used in the analyses presented herein, but additional available datasets are also utilized. The P-3 released several of the Vaisala RD94 GPS dropsondes during its eyewall penetrations. These dropsondes provide PTH and velocity observations along a trajectory as they fall from the aircraft with a $2-\mathrm{Hz}$ sampling frequency, resulting in a vertical resolution of approximately $5 \mathrm{~m}$. RD94 dropsondes are quality controlled using the Atmospheric Sounding Processing Environment (ASPEN) software. In situ flight-level kinematic and thermodynamic observations are collected by the $\mathrm{P}-3$ instrumentation at a $1-\mathrm{Hz}$ sampling frequency. A correction is applied to both the temperature and dewpoint temperature observations to account for potential instrument wetting events following the method outlined by Zipser et al. (1981) and discussed by Eastin et al. (2002). Atmospheric motion vectors (AMVs) derived from feature tracking in satellite imagery through a combination of water vapor and infrared channels are obtained from the Cooperative Institute for Meteorological Satellite Studies (CIMSS) and also included in the analyses (Velden et al. 1997, 2005).

Observations are analyzed for each IOP using a twodimensional (axisymmetric) variational analysis technique called Spline Analysis at Mesoscale Utilizing Radar and Aircraft Instrumentation (SAMURAI; Bell et al. 2012; Foerster et al. 2014; Foerster and Bell 2017). Given a set of observations and associated error estimates, the SAMURAI technique yields the maximum likelihood estimate of the atmosphere through a minimization of a variational cost function. Unique characteristics of the SAMURAI technique include the ability to perform the analysis directly in an axisymmetric cylindrical coordinate system and the absence of any imposed balance or physical constraints other than mass continuity. The SAMURAI technique utilizes a finite element approach, which employs second-order continuous cubic B-splines as a set of basis functions (Ooyama 2002), providing high numerical precision for derived quantities.

from (a) the Special Sensor Microwave Imager (SSM/I) aboard DMSP F15 at 0934 UTC 22 Oct, (b) the SSMIS aboard DMSP F19 at 1359 UTC 23 Oct, and (c) the Advanced Microwave Scanning Radiometer 2 (AMSR2) aboard GCOM-W1 at 1911 UTC 23 Oct. Source: Naval Research Laboratory. 
(a) $\mathrm{RI}(r, \phi)$

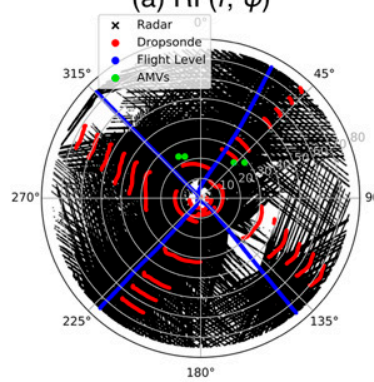

(c) $\mathrm{MI}(r, \phi)$

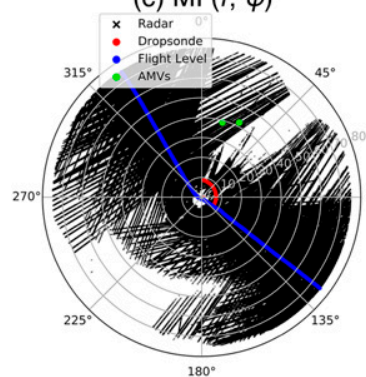

(e) $\operatorname{RW}(r, \phi)$

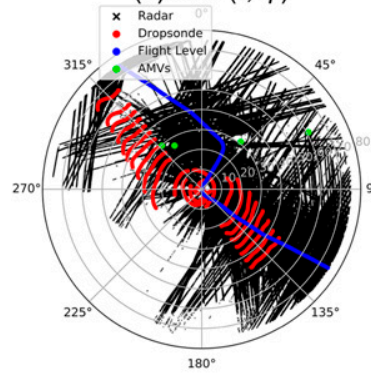

(b) $\mathrm{RI}(r, z)$

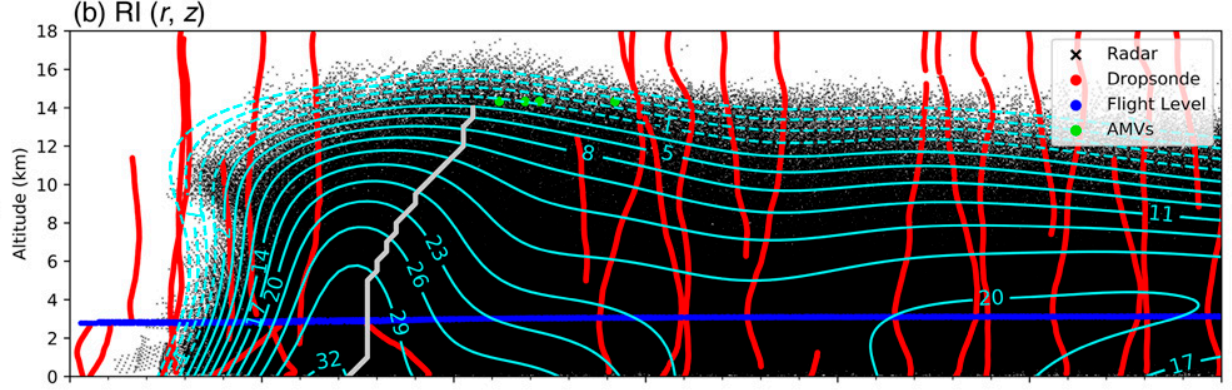

(d) $\mathrm{MI}(r, z)$
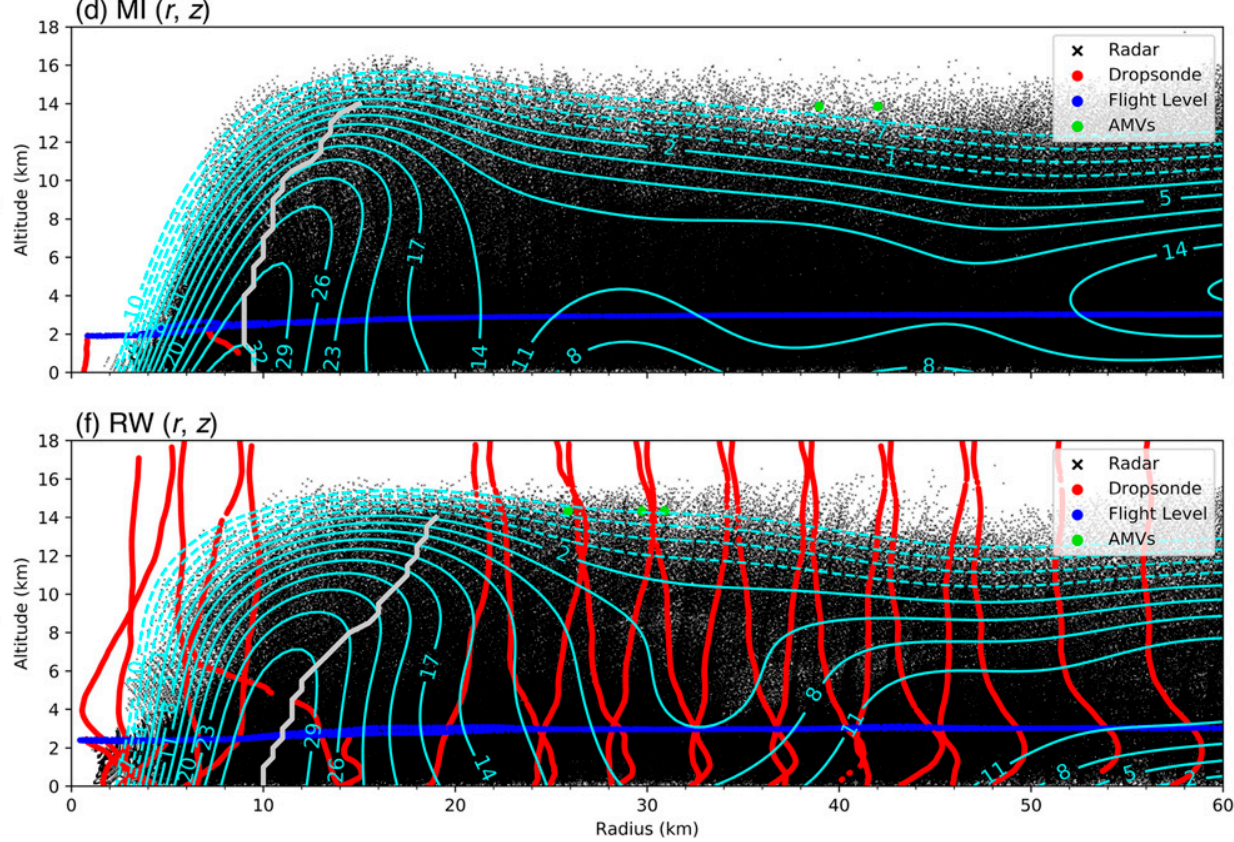

FIG. 3. Storm-relative data distributions for each intensive observing period (IOP) in (a),(c),(e) the radius-azimuth ( $r, \phi)$ plane and (b),(d),(f) the radius-height $(r, z)$ plane. Dropsonde trajectories (XDDs and RD94 sondes) are shown in red, in situ measurements from the P-3 in blue, AMVs in green, and P-3 tail Doppler radar observation points in black. Radar reflectivity (cyan, contoured every $3 \mathrm{~dB} Z$ ) and the RMW (gray) from each respective SAMURAI analysis are overlaid in (b), (d), and (f).

Axisymmetric cylindrical SAMURAI analyses are created for each respective IOP using circulation centers identified as the point that maximizes the tangential circulation within the RMW (Marks et al. 1992). The 2-8-km-layer-averaged circulation center is specified as the center of circulation for each IOP analysis, and an $f$-plane approximation is assumed with the Coriolis parameter calculated at this location. Figure 3 shows the data distribution for each IOP in both polar and axisymmetric coordinates, with the radar reflectivity overlaid in cyan contours for the axisymmetric analyses. All axisymmetric cross sections presented herein are scaled to preserve the aspect ratio. The Doppler radar observations provide thorough coverage throughout the analysis domains, giving high confidence in the retrieved kinematic fields except in the $5-\mathrm{km}$ radial region nearest to the axis because of the lack of hydrometeors in the eye. A soft $w=0$ (vertical velocity) variational constraint is applied in clear air with a standard deviation of $0.5 \mathrm{~m} \mathrm{~s}^{-1}$ to reduce numerically spurious vertical velocities at small radii. As a result, the magnitude of downdrafts in Patricia's eye may be underestimated. XDDs released by the WB-57 (red dots in Fig. 3) extend throughout the full depth of the troposphere and provide extensive radial coverage except near the RMW where a few sondes failed before reaching the surface.

Because of the different sampling resolutions of the dropsondes and Doppler radar, separate analyses are created for the thermodynamic fields and kinematic fields. SAMURAI uses a spline-cutoff (Ooyama 2002) and recursive Gaussian filter (Purser et al. 2003) to smooth the analyses and spread both kinematic and thermodynamic information across the analysis domain. 
(a) Tangential Velocity (shaded, $\mathrm{m} \mathrm{s}^{-1}$ ), Transverse Circulation (vectors, $\mathrm{m} \mathrm{s}^{-1}$ )

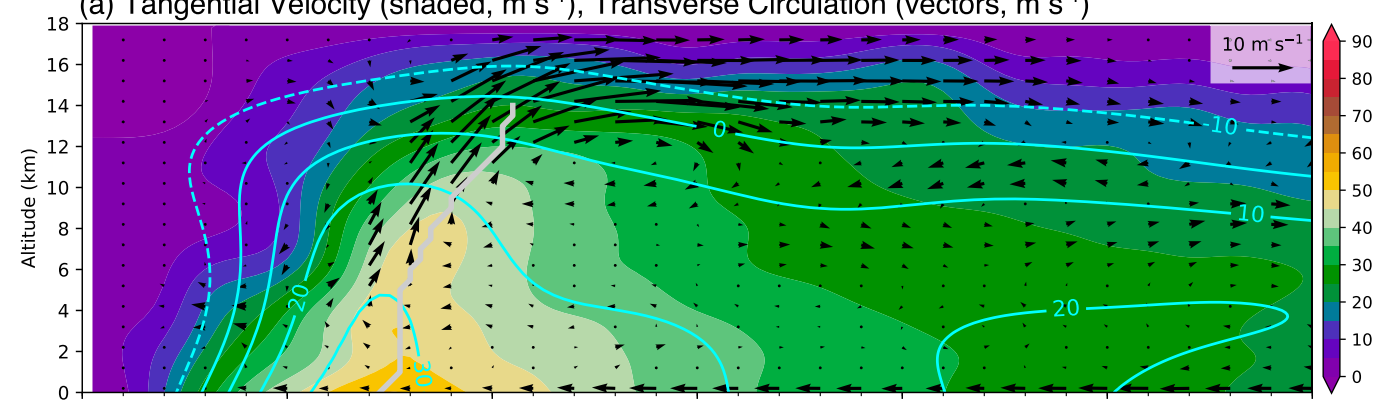

(b) Potential temperature (shaded, K), Absolute Angular Momentum (contoured, $10^{6} \mathrm{~m}^{2} \mathrm{~s}^{-1}$ )

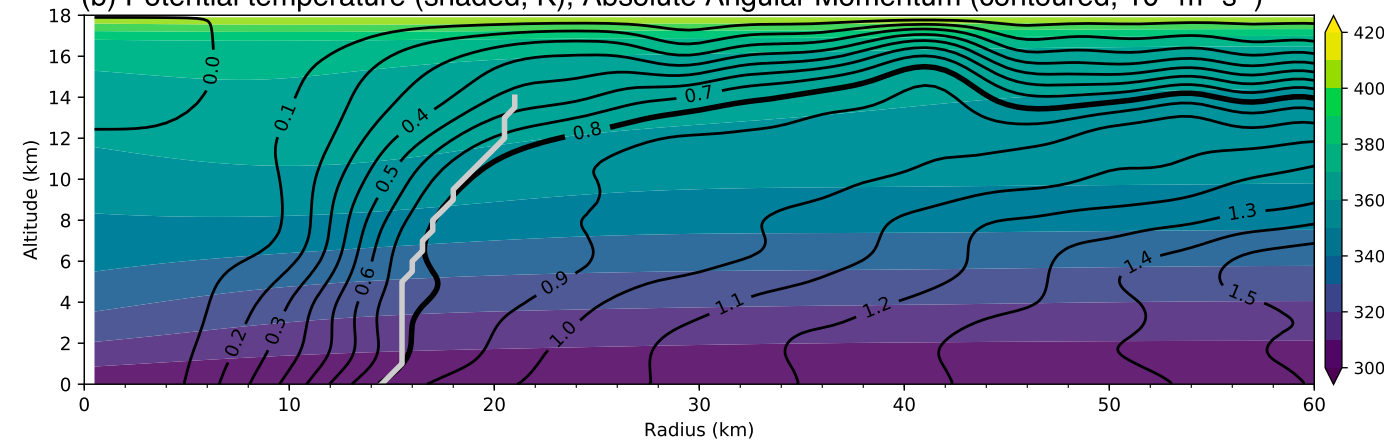

FIG. 4. Axisymmetric SAMURAI analysis for the RI IOP in Hurricane Patricia (1715-1915 UTC 22 Oct 2015). (a) Tangential velocity (shaded, $\mathrm{m} \mathrm{s}^{-1}$ ) is overlaid with the transverse circulation (vectors, $\mathrm{m} \mathrm{s}^{-1}$ ) and radar reflectivity (cyan, contoured every $10 \mathrm{dBZ}$ ). (b) Potential temperature (shaded, $\mathrm{K}$ ) is overlaid with absolute angular momentum (black, contoured every $10^{6} \mathrm{~m}^{2} \mathrm{~s}^{-1}$ ). The angular momentum surface given by $0.8 \times 10^{6} \mathrm{~m}^{2} \mathrm{~s}^{-1}$ is shown in bold. In both panels, the RMW is shown in gray.

Tests with different spatial resolutions and filter lengths indicate trade-offs between the level of detail, the magnitude of resolved gradients, and potential analysis artifacts resulting from the different sampling resolutions. The analyses presented herein limit resolved scales to 6 and $0.5 \mathrm{~km}$ in the radial and vertical directions, respectively, for the thermodynamic analyses, and 1.5 and $0.5 \mathrm{~km}$ in the radial and vertical directions, respectively, for the kinematic analyses. Since several of the XDDs failed in the upper troposphere near the eyewall, the spatial filters interpolate the observations across an approximately $10-20-\mathrm{km}$ data gap near the eyewall for the thermodynamic analysis of each IOP. A higher level of detail is preserved in the kinematic analyses given the dense Doppler radar sampling.

Last, we note that the WB-57 overpass on 23 October coincided with the RW IOP (second center pass of the P-3) and not the MI IOP, which is evidenced by the lack of dropsondes in the data distribution for the MI IOP (Figs. 3c,d). To resolve this issue, we assume that the axisymmetric thermodynamic structure evolves on a slow enough time scale to warrant using the same thermodynamic analysis for both the MI and RW IOPs. This assumption will be examined in more detail in the discussion section.

\section{Axisymmetric structural evolution}

\section{a. Rapid intensification}

On 22 October, Hurricane Patricia entered an environment characterized by sea surface temperatures in excess of $30^{\circ} \mathrm{C}$ (Fig. 1b) with weak deep-layer (850-200 hPa) environmental vertical wind shear (not shown). ${ }^{1}$ These favorable environmental conditions allowed Patricia to rapidly intensify from a tropical storm $\left(\sim 30 \mathrm{~m} \mathrm{~s}^{-1}\right)$ at 0000 UTC to a category 4 major hurricane $\left(\sim 60 \mathrm{~m} \mathrm{~s}^{-1}\right)$ at $1800 \mathrm{UTC}$, at which point it was sampled by both the P-3 and WB-57 (see Fig. 1).

The axisymmetric kinematic and thermodynamic structure of Patricia for the RI IOP (1715-1915 UTC 22 October) is shown in Fig. 4. At this stage, Patricia is

\footnotetext{
${ }^{1}$ We note here that all references to the deep-layer $(850-200 \mathrm{hPa})$ environmental vertical wind shear magnitude and direction are obtained from the Statistical Hurricane Intensity Prediction Scheme (SHIPS) predictors labeled "SHDC" and "SDDC." SHDC and SDDC represent the 850-200-hPa vertical wind shear magnitude and direction, respectively, averaged between 0 - and $500-\mathrm{km}$ radii from the vortex center after removing the vortex circulation. These variables are obtained from the operational Global Forecast System (GFS) analyses.
} 
(a) Absolute Vorticity (shaded, $\left.10^{-3} \mathrm{~s}^{-1}\right), \partial \theta / \partial \mathrm{z}$ (contoured, $\mathrm{K} \mathrm{km}^{-1}$ )

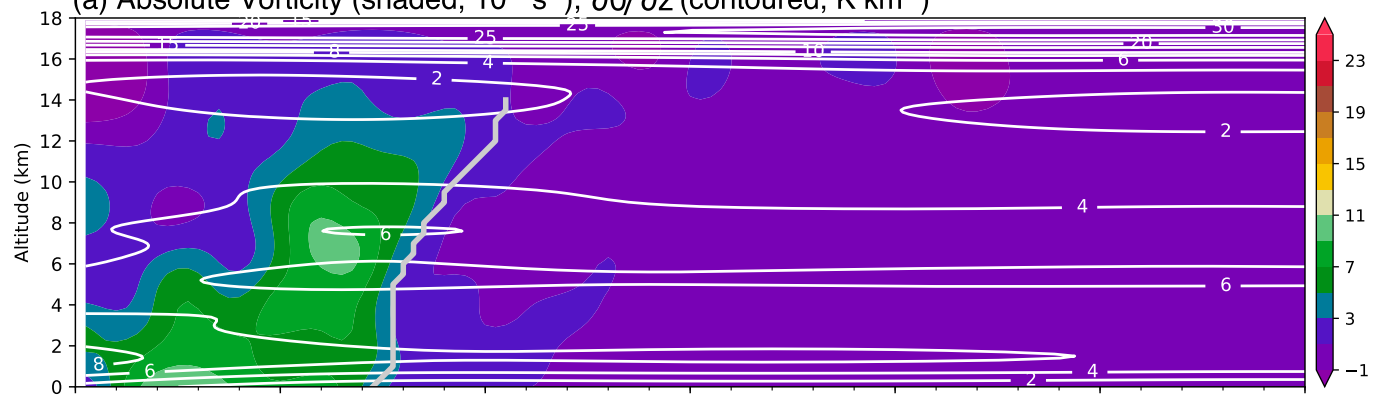

(b) Dry Potential Vorticity (PVU, $10^{-6} \mathrm{~K} \mathrm{~kg}^{-1} \mathrm{~m}^{2} \mathrm{~s}^{-1}$ ), Transverse Circulation (vectors, $\mathrm{m} \mathrm{s}^{-1}$ )

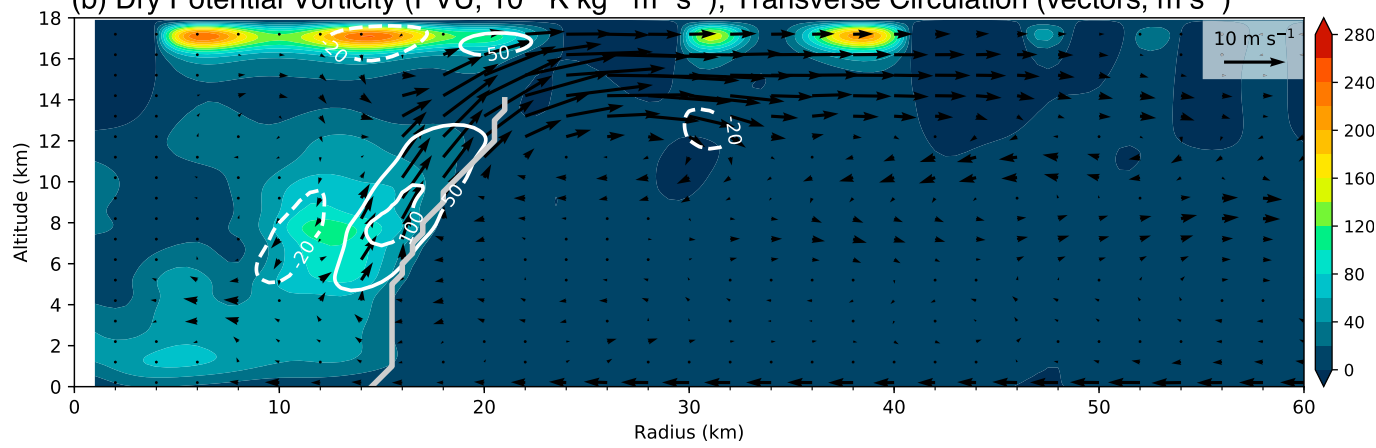

FIG. 5. RI axisymmetric SAMURAI analysis in Hurricane Patricia (1715-1915 UTC 22 Oct 2015). (a) Absolute vertical vorticity $\eta$ (shaded, $10^{-3} \mathrm{~s}^{-1}$ ) is overlaid with the vertical gradient of potential temperature (white, contoured every $2 \mathrm{~K} \mathrm{~km}^{-1}$ between 0 and $10 \mathrm{~K} \mathrm{~km}^{-1}$ and every $5 \mathrm{~K} \mathrm{~km}^{-1}$ between 10 and $30 \mathrm{~K} \mathrm{~km}^{-1}$ ). (b) Dry Ertel's PV (shaded, PVU) is overlaid with the transverse circulation (vectors, $\mathrm{m} \mathrm{s}^{-1}$ ) and the diabatic heating $\dot{\theta}$ estimate (white, contours at $-20,50,100,150$, and $200 \mathrm{~K} \mathrm{~h}^{-1}$ ). In both figures, the RMW is shown in gray.

characterized by a remarkably compact circulation with an RMW at 2-km altitude of $15 \mathrm{~km}$. Deep convection (shown by the cyan contours representing radar reflectivity) is concentrated radially inward of the RMW, denoted by the gray line, which is a configuration commonly observed with intensifying TCs (e.g., Rogers et al. 2013a). A secondary reflectivity maximum is apparent at a radius of $\sim 45 \mathrm{~km}$ and is associated with a pronounced principal rainband evident at this stage in Patricia's development (Fig. 2). The deep, cyclonic primary circulation near the eyewall has tangential velocities exceeding $30 \mathrm{~ms}^{-1}$ at $12-\mathrm{km}$ altitude (Fig. 4a), with a relatively small slope between 2 - and 8 -km altitudes. The secondary circulation is characterized by a shallow inflow layer that penetrates radially inward of the RMW, accelerates upward in the eyewall updraft, and then turns radially outward to a strong outflow $\left(>10 \mathrm{~m} \mathrm{~s}^{-1}\right)$ between 12 - and 16-km altitudes.

Figure $4 \mathrm{~b}$ illustrates the potential temperature structure of Patricia for the RI IOP in shading, overlaid by surfaces of absolute angular momentum given by $M=r v+(1 / 2) f r^{2}$, where $f$ is the Coriolis parameter, and $v$ is the axisymmetric tangential velocity. The contour for $M=0.8 \times 10^{6} \mathrm{~m}^{2} \mathrm{~s}^{-1}$ is shown in bold to emphasize the evolution of $M$ near the RMW throughout the three IOPs. The potential temperature contours descend toward the axis of rotation as Patricia's warm core develops during RI, and the $M$ surfaces are tightly packed radially inward of the RMW, contributing to an intense vorticity tower (Fig. 5a). The axisymmetric absolute vertical vorticity is given by $\eta=f+\partial(r v) / r \partial r$. Patricia's compact primary circulation contributes to both strong curvature $(v / r)$ and radial shearing $(\partial v / \partial r)$ components of the vorticity, with $\eta$ values between 5 and $10 \times 10^{-3} \mathrm{~s}^{-1}$ found along the inner edge of the RMW.

In addition to absolute vorticity, Fig. 5a shows contours of the vertical gradient of potential temperature $\theta$, which is related to the static stability. Patricia's thermodynamic structure is characterized by a local static stability maximum in the eye at approximately $2-\mathrm{km}$ altitude where a low-level inversion is often observed, separating the dry, subsiding air aloft from relatively cooler and moister air below (Jordan 1961; Franklin et al. 1988; Willoughby 1998). The high-altitude flight level of the WB-57 provided thermodynamic observations in the upper-troposphere-lower-stratosphere (UTLS) region of Patricia, showing strong stratification between 16- and $18-\mathrm{km}$ altitudes and a vertical gradient of $\theta$ exceeding $25 \mathrm{~K} \mathrm{~km}^{-1}$. 
The kinematic and thermodynamic analyses allow for calculation of the full-tropospheric, axisymmetric PV in Patricia without the use of balance assumptions. Ertel's $\mathrm{PV}^{2}$ is given in geometric, axisymmetric cylindrical coordinates as

$$
P=\frac{1}{\rho}\left\{-\frac{\partial v}{\partial z} \frac{\partial \theta}{\partial r}+\left[f+\frac{\partial(r v)}{r \partial r}\right] \frac{\partial \theta}{\partial z}\right\}
$$

where $\rho$ is the dry air density and the remaining variables assume their aforementioned definitions. Figure $5 \mathrm{~b}$ shows the axisymmetric PV structure of Patricia during RI, overlaid by the transverse circulation vectors. Patricia possesses a hollow-tower PV structure with a midlevel maximum located in the eyewall exceeding 100 PV units (PVU; 1 PVU $=10^{-6} \mathrm{~K} \mathrm{~kg}^{-1} \mathrm{~m}^{2} \mathrm{~s}^{-1}$ ). A second local maximum of $\sim 60 \mathrm{PVU}$ is evident in the low-level eye, collocated with the local maximum in static stability (Fig. 5a). In the UTLS region, high static stability leads to several PV rings with maxima on the order of 200 PVU. Given that observations in the UTLS are sparse, there is a high degree of uncertainty in the magnitude of these PV rings. However, given both the strong rotation and static stability in this region, we believe the analyzed PV rings are at least qualitatively realistic.

An estimate for Patricia's diabatic heating $\dot{\theta}$ is shown in white contours, computed under an approximate steady-state assumption where diabatic heating is exactly offset by adiabatic cooling (e.g., Roux et al. 1993). We expect the local tendency of potential temperature $(\partial \theta / \partial t)$ in the eyewall region to be a small residual between latent heating and adiabatic cooling that is less than $\sim 2 \mathrm{~K} \mathrm{~h}^{-1}$ (Zhang et al. 2002; Stern and Zhang 2013). If we assume the local tendency is exactly zero, the diabatic heating can be estimated as $\dot{\theta}=u \partial \theta / \partial r+$ $w \partial \theta / \partial z$, where $u$ and $w$ represent the radial and vertical velocities, respectively. We note that heating from asymmetric eddy sources and additional diabatic processes such as radiation are not included in this heating estimate. The absolute uncertainty in the calculated heating magnitude is difficult to estimate because of uncertainties in the analysis and unresolved processes, such that we limit the interpretation of the heating field to a qualitative discussion of the location and relative magnitudes of the heating in each IOP. During the

\footnotetext{
${ }^{2}$ In this study, we use the original "dry" definition of PV with potential temperature serving as the relevant thermodynamic variable. A PV principle valid for a moist, precipitating atmosphere has been derived (Schubert et al. 2001; Schubert 2004), but given uncertainties in the measured humidity and quantitative similarity between the moist and dry PV, we have opted to use the standard PV definition.
}

RI period, heating is maximized at midlevels radially inward of the RMW and slightly outward of the PV maximum (Fig. 5). As will be shown, subsequent changes in the heating and PV fields have a close correspondence.

\section{b. Maximum intensity}

Hurricane Patricia continued to intensify, and reached its lifetime maximum intensity of $95 \mathrm{~m} \mathrm{~s}^{-1}$ at $1200 \mathrm{UTC}$ 23 October. Patricia remained in an environment with sea surface temperatures in excess of $30^{\circ} \mathrm{C}$ (Fig. 1b); however, it began to encounter weak to moderate deeplayer vertical wind shear between $\sim 4$ and $5 \mathrm{~m} \mathrm{~s}^{-1}$ from the south-southwest (not shown). The MI IOP took place between 1710 and 1800 UTC 23 October, approximately $6 \mathrm{~h}$ after Patricia reached its maximum lifetime intensity. Patricia maintained category 5 major hurricane status at this time with an intensity of $\sim 93 \mathrm{~m} \mathrm{~s}^{-1}$. Figure 6 shows the axisymmetric kinematic and thermodynamic structure of Patricia for the MI IOP. In the 24-h time window separating RI and MI, Patricia's RMW contracted to $9 \mathrm{~km}$ at $2-\mathrm{km}$ altitude and the slope of the RMW between 2- and 8-km altitudes decreased. A decreased slope of the RMW with altitude in correspondence with an overall contraction of the RMW is consistent with previous findings (Stern and Nolan 2009; Stern et al. 2014). Deep convection persisted in the primary eyewall and a secondary reflectivity maximum is apparent near a radius of $60 \mathrm{~km}$ (Fig. 3b), likely associated with deep convection in the principal rainband wrapping around the system (Fig. 2b). Although the RMW is nearly vertically aligned, the convection in the primary eyewall possesses a larger slope such that its core is displaced radially outward of the primary eyewall above 4-km altitude (Fig. 6a).

Patricia's kinematic structure during MI is characterized by a deep, intense primary circulation with tangential velocities exceeding $60 \mathrm{~m} \mathrm{~s}^{-1}$ at $12-\mathrm{km}$ altitude. The inflow layer deepened and intensified compared to the RI IOP, penetrating radially inward of the RMW where strong convergence is located at the base of the eyewall. A midlevel inflow layer has developed near the eyewall between $\sim 2$ - and $4-\mathrm{km}$ altitudes, appearing to be somewhat separated from the near-surface inflow layer. Updrafts concentrated along the RMW approach magnitudes of $20 \mathrm{~m} \mathrm{~s}^{-1}$ near $8-\mathrm{km}$ altitude, turning radially outward to an intensified outflow that exceeds $15 \mathrm{~m} \mathrm{~s}^{-1}$ between $14-$ and $16-\mathrm{km}$ altitudes.

As described in the methods section, we use the thermodynamic structure from the WB-57 pass $3 \mathrm{~h}$ later (during the RW IOP) to analyze the MI IOP. Figure 6b shows the potential temperature structure of Patricia 


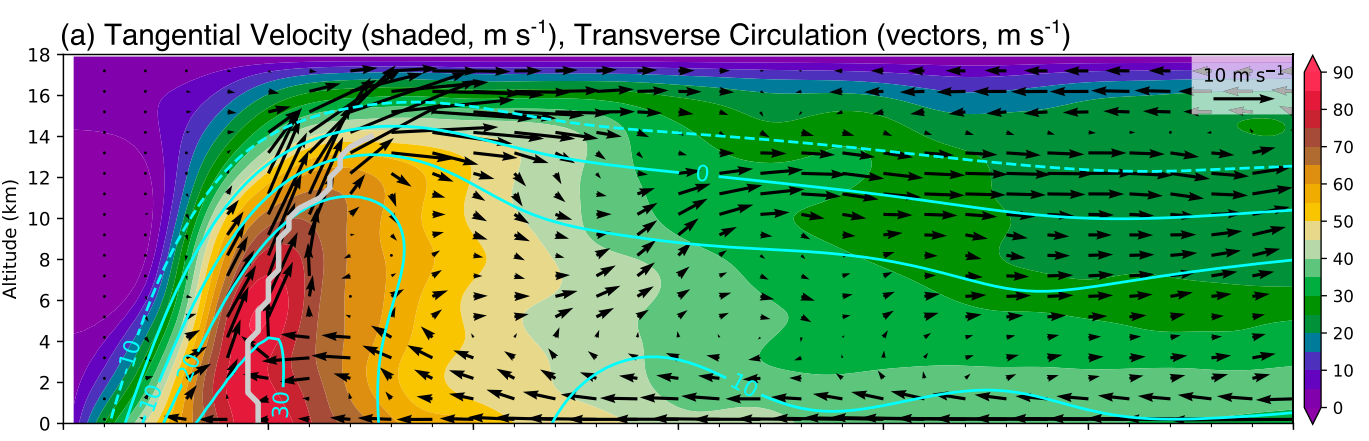

(b) Potential temperature (shaded, K), Absolute Angular Momentum (contoured, $10^{6} \mathrm{~m}^{2} \mathrm{~s}^{-1}$ )

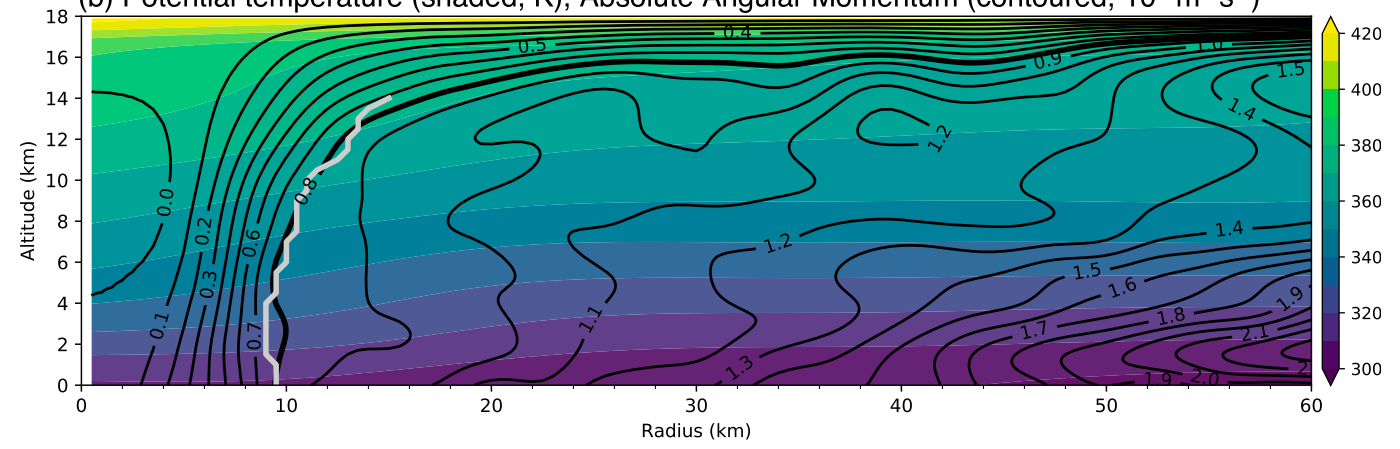

FIG. 6. As in Fig. 4, but for the MI IOP axisymmetric SAMURAI analysis in Hurricane Patricia (1710-1800 UTC 23 Oct 2015). Note that the thermodynamic analysis for the MI IOP was created using data that coincided with the RW IOP.

evaluated for these two IOPs, along with the $M$ surfaces unique to the MI IOP. Interestingly, though the warm core has intensified during the $\sim 24 \mathrm{~h}$ between RI and RW, the static stability is largely the same (cf. Figs. 5a and 9a). The contour for $M=0.8 \times 10^{6} \mathrm{~m}^{2} \mathrm{~s}^{-1}$ has migrated radially inward, remaining mostly aligned with the RMW from the surface up to nearly $14-\mathrm{km}$ altitude, and the radial gradient of the $M$ surfaces has increased inward of the RMW. Figure 7a shows Patricia's remarkable vorticity structure resulting from its intensification and contraction between RI and MI. The radial shear of the tangential velocity approaches values of $9 \mathrm{~m} \mathrm{~s}^{-1} \mathrm{~km}^{-1}$ between the axis of rotation and RMW (Fig. 6a), contributing to $\eta$ exceeding $25 \times 10^{-3} \mathrm{~s}^{-1}$ radially inward of the RMW.

A local static stability maximum remains in the lowlevel eye with a similar magnitude to that observed during RI, and the upper portion of the outflow layer in the UTLS remains highly stratified. Figure $7 b$ shows that a significant evolution has occurred from the RI period as the hollow tower of PV has further concentrated into an intense, vertically coherent tower. The hollow tower is characterized by PV values up to 250 PVU located along the inner edge of the RMW and extending from near the surface up to $12-\mathrm{km}$ altitude. Local PV maxima are again noted in the UTLS region with amplitudes exceeding 250 PVU near the eyewall and $\sim 160$ PVU radially outward of the RMW. Interestingly, a UTLS PV patch located at approximately $r=45 \mathrm{~km}$ is in the region where Patricia's secondary eyewall eventually develops, but it is unclear whether this is coincidental or dynamically relevant, especially given uncertainties in the magnitude of $\mathrm{PV}$ in the UTLS region.

\section{c. Rapid weakening}

Approximately $3 \mathrm{~h}$ after MI, the P-3 flew its second center pass through Patricia between 2015 and 2100 UTC 23 October, denoted herein as the RW IOP. Patricia remained in a region with high sea surface temperatures, exceeding $31^{\circ} \mathrm{C}$. The magnitude of the deep-layer vertical wind shear was increasing from $\sim 5 \mathrm{~m} \mathrm{~s}^{-1}$ at 1800 UTC 23 October to $\sim 13 \mathrm{~m} \mathrm{~s}^{-1}$ at 0000 UTC 24 October. Figure 8a shows the axisymmetric kinematic and thermodynamic structure of Patricia for the RW IOP. Although MI and RW are separated by only $3 \mathrm{~h}$, a dramatic structural change is evident in the analyses. Deep convection is concentrated along the RMW, but now a pronounced secondary reflectivity maximum is located between a radius of 45 and $60 \mathrm{~km}$, associated with a developing secondary eyewall. The two eyewalls are separated by a region of relatively low reflectivity values known as the "moat" (Houze et al. 2007). 

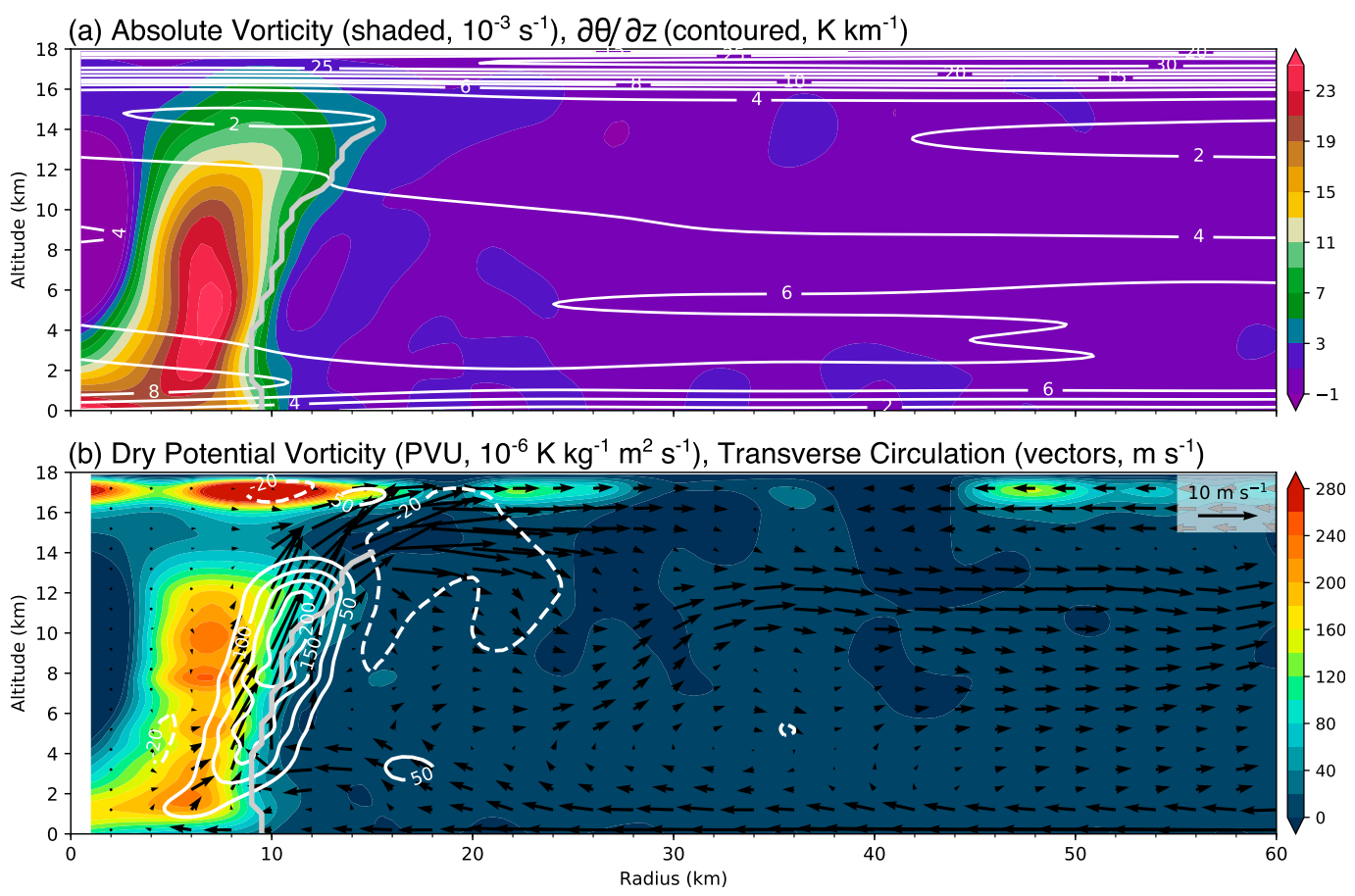

FIG. 7. As in Fig. 5, but for the MI IOP axisymmetric SAMURAI analysis. Note that the thermodynamic analysis for the MI IOP was created using data that coincided with the RW IOP.

The slope of the RMW has increased significantly and the tangential velocity maximum is now located near $5-\mathrm{km}$ altitude (Fig. 8). The secondary eyewall is apparent in both the primary and secondary circulations with an overall shallower structure compared to the primary eyewall. The near-surface inflow spiraling into the primary eyewall appears to have been impeded by the formation of the secondary eyewall, while the midlevel inflow has developed a toroidal circulation around $5-\mathrm{km}$ altitude. We note that this toroidal circulation is vertically centered near the freezing level, suggesting it may be related to strong diabatic heating at this altitude. The magnitude of the updrafts in the primary and secondary eyewalls are comparable with maximum values of $\sim 5 \mathrm{~m} \mathrm{~s}^{-1}$. The outflow associated with the primary eyewall has weakened to $\sim 10 \mathrm{~m} \mathrm{~s}^{-1}$ while the outflow associated with the secondary eyewall has a maximum of $\sim 5 \mathrm{~m} \mathrm{~s}^{-1}$.

In addition to the increased slope of the RMW, the radial shear of tangential velocity between the axis of rotation and the RMW has decreased. The $M$ surfaces have spread out radially in the primary eyewall, especially below 4-km altitude where the inflow has been reduced (Fig. 8b). In contrast, the $M$ surfaces have converged radially in the developing secondary eyewall. The changes in tangential velocity and absolute angular momentum are manifested in the absolute vorticity structure as an overall decrease in the magnitude of vorticity within the primary eyewall, with maximum values on the order of
$15 \times 10^{-3} \mathrm{~s}^{-1}$ (Fig. 9a). The PV structure during RW no longer resembles a pronounced hollow tower but rather a more diffuse, vertically sloping structure with two separate maxima at midlevels (Fig. 9b). PV is no longer concentrated along the inner edge of the RMW and maximum values have decreased to $\sim 120$ PVU. The local maximum in PV is collocated with the local static stability maximum in the low-level eye rather than extending throughout the full troposphere. This PV structure suggests substantial mixing at the eyeeyewall interface has occurred, which will be discussed further in the following section. It is worth emphasizing that these dramatic inner-core structural changes took place on a short time scale of approximately $3 \mathrm{~h}$.

\section{Potential vorticity evolution}

To gain further insight into Patricia's rapid intensification and weakening, we examine the evolution of the axisymmetric PV field in more detail. We begin by noting that the estimates of PV presented herein are limited in their quantitative accuracy because of observational uncertainties, undersampling, and spatial and temporal filtering. Furthermore, we use the axisymmetric thermodynamic analysis valid for the RW IOP to compute PV in the MI IOP under the assumption that the axisymmetric thermodynamic structure evolves on a slower time scale than the axisymmetric wind field. 


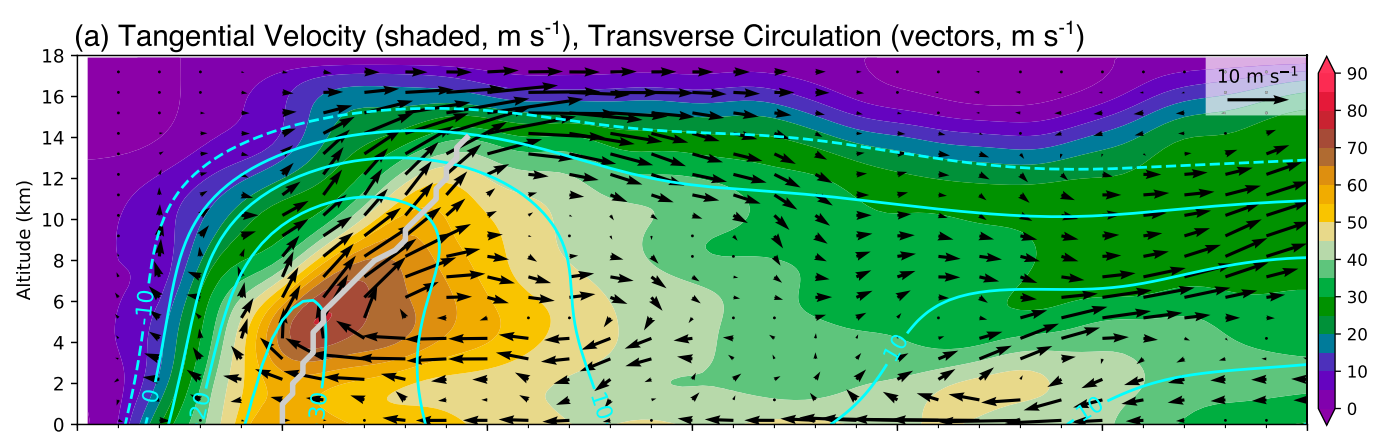

(b) Potential temperature (shaded, K), Absolute Angular Momentum (contoured, $10^{6} \mathrm{~m}^{2} \mathrm{~s}^{-1}$ )

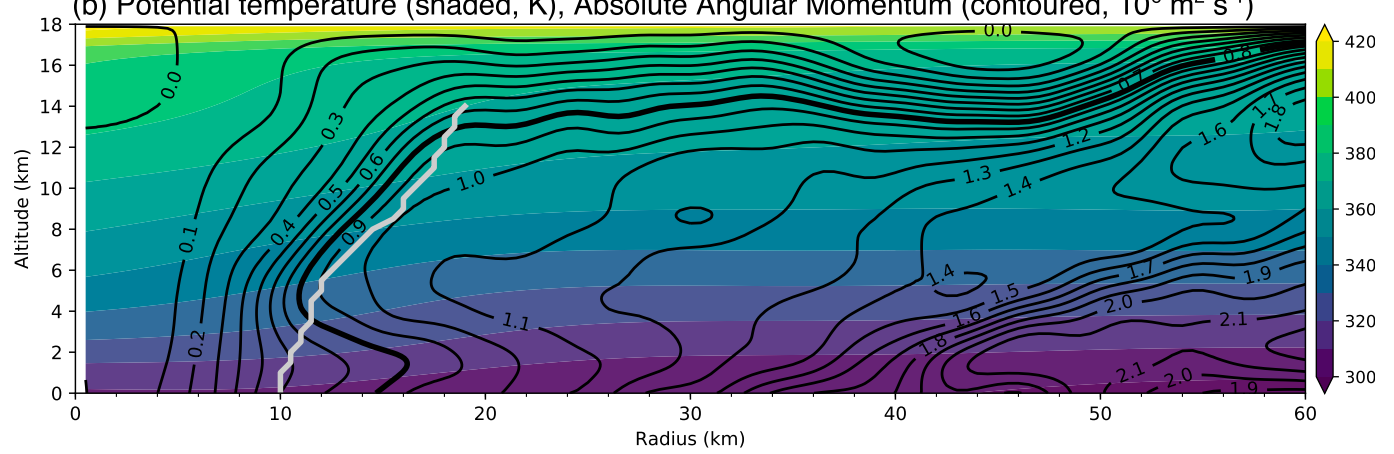

FIG. 8. As in Fig. 4, but for the RW IOP axisymmetric SAMURAI analysis in Hurricane Patricia (2015-2100 UTC 23 Oct 2015).

Figures $7 \mathrm{~b}$ and $9 \mathrm{~b}$ demonstrate that Patricia experienced a dramatic PV structural evolution in the $3 \mathrm{~h}$ separating the MI and RW IOPs. However, given the remarkably similar vertical gradients of potential temperature between the RI and RW IOPs (cf. Figs. 5a and 9a), we posit that Patricia's $\mathrm{PV}$ evolution between MI and RW is largely dominated by vorticity evolution with changes in the mass field contributing to a lesser degree. Despite these limitations and assumptions, the high-resolution analyses from the TCI observations yield quantitative estimates of the PV evolution, which provide a useful framework for understanding Patricia's rapid intensification and weakening.

\section{a. Coordinate transformation}

Seminal work by Schubert and Hack (1983) introduced the transformed Eliassen balanced vortex model in which absolute angular momentum served as an independent variable, simplifying the transverse circulation equation and providing a useful way to obtain dynamical insight into the evolution of TCs. The potential radius $R$ is defined for an inertially stable vortex as $(1 / 2) f R^{2}=r v+(1 / 2) f r^{2} \equiv M$, and represents the radius to which a parcel must be displaced (conserving absolute angular momentum) in order for its tangential velocity $v$ to become zero. The Lagrangian evolution of $R$ is therefore related to sources and sinks of $M$, and is given by

$$
f R \dot{R}=\dot{M}=-\overline{u^{\prime} \frac{\partial M^{\prime}}{\partial r}}-\overline{w^{\prime} \frac{\partial M^{\prime}}{\partial z}},
$$

where $\dot{R}=D R / D t, \dot{M}=D M / D t$, and the material derivative operator is given by $D / D t=\partial / \partial t+u \partial / \partial r+$ $w \partial / \partial z$. The terms on the right-hand side of Eq. (2) denote the eddy sources and sinks of absolute angular momentum (torques), which can arise from frictional fluid stresses and asymmetric processes.

A notable advantage of the potential radius framework is the natural coordinate stretching it provides in regions where the vertical vorticity exceeds the planetary vorticity [see Eqs. (8) and (10) of Schubert and Hack (1983)]. Schubert and Alworth (1987) took an additional step toward simplifying the transformed Eliassen balanced vortex model by simultaneously using potential radius and isentropic (potential temperature) coordinates. Following the derivation laid out in section 2c of Schubert and Alworth (1987) and section 4 of Hausman et al. (2006), the Lagrangian PV equation for a dry axisymmetric vortex can be written in the $(R, \theta)$ coordinate space as

$$
\frac{D P}{D t}=P\left[\frac{\partial(R \dot{R})}{R \partial R}+\frac{\partial \dot{\theta}}{\partial \theta}\right],
$$

where the material derivative operator is given by 
(a) Absolute Vorticity (shaded, $10^{-3} \mathrm{~s}^{-1}$ ), $\partial \theta / \partial \mathrm{z}$ (contoured, $\mathrm{K} \mathrm{km}^{-1}$ )

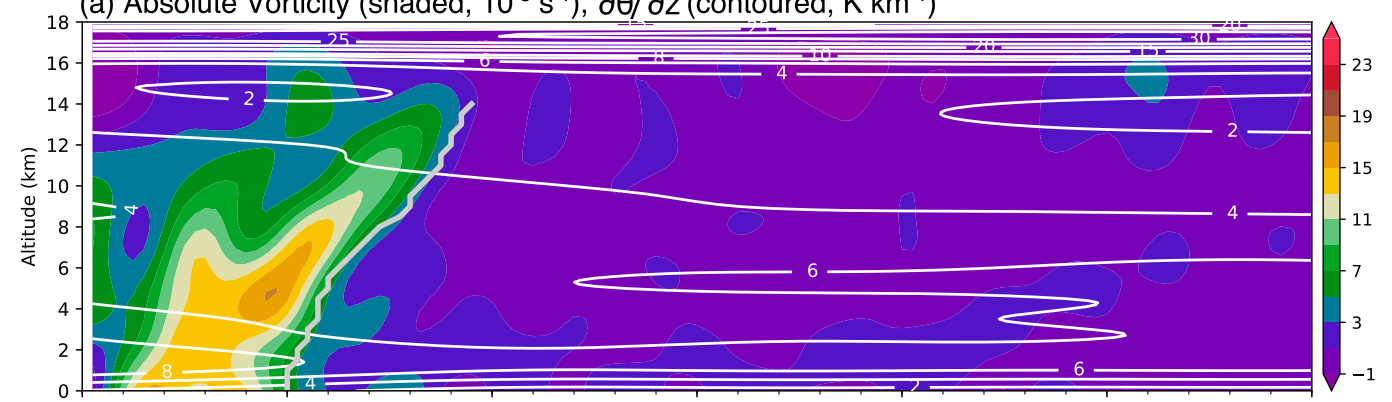

(b) Dry Potential Vorticity (PVU, $10^{-6} \mathrm{~K} \mathrm{~kg}^{-1} \mathrm{~m}^{2} \mathrm{~s}^{-1}$ ), Transverse Circulation (vectors, $\mathrm{m} \mathrm{s}^{-1}$ )

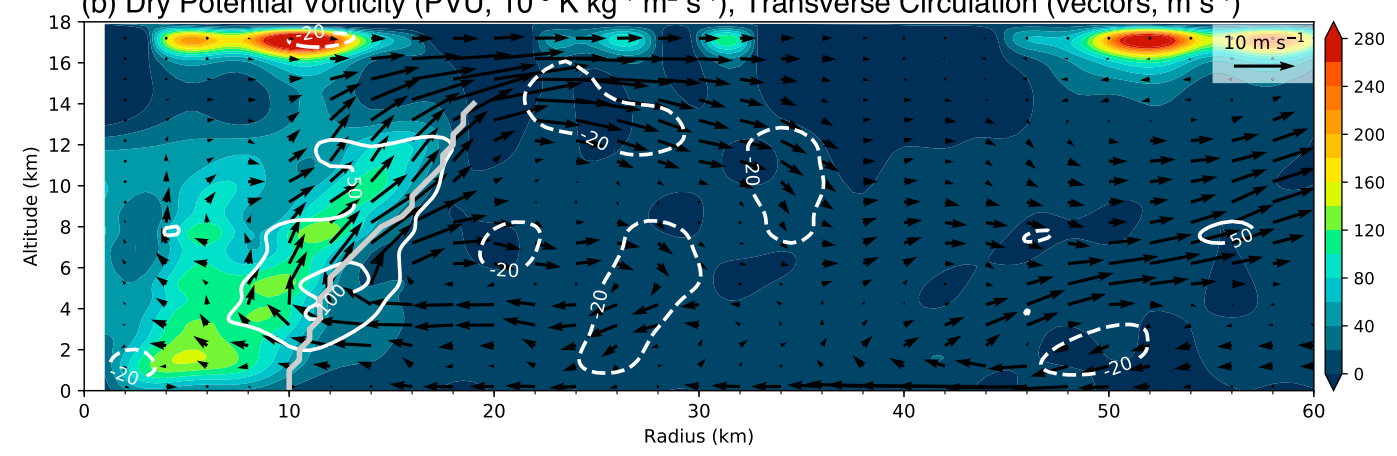

FIG. 9. As in Fig. 5, but for the RW IOP axisymmetric SAMURAI analysis.

$$
\frac{D}{D t}=\frac{\partial}{\partial \tau}+\dot{R} \frac{\partial}{\partial R}+\dot{\theta} \frac{\partial}{\partial \theta} .
$$

In this coordinate space, $\tau$ is equivalent to time $t$, but $\partial / \partial \tau$ implies fixed $(R, \theta)$ compared to a fixed $(r, z)$ position given by $\partial / \partial t$. Adopting the $(R, \theta)$ coordinate space allows analysis of Patricia's PV structure from a dynamically relevant perspective in which "movement" is due to sources and sinks of absolute angular momentum and diabatic processes, which can concentrate or dilute PV within isentropic layers (Haynes and McIntyre 1987). In this coordinate space, radial movement across $R$ surfaces is associated with material changes in the absolute angular momentum of an air parcel through eddy torques $\dot{R}$ [see Eq. (2)], and vertical movement across $\theta$ surfaces is associated with material changes in the potential temperature of an air parcel through diabatic processes $\dot{\theta}$. The material loss or gain of PV is then associated with both gradients in $\dot{R}$ and $\dot{\theta}$, or "acceleration" across $M$ and $\theta$ surfaces in the transformed coordinate space given by the two terms on the right-hand side of Eq. (3), respectively.

\section{b. Patricia's evolution in $(R, \theta)$ space}

Figure 10 shows Patricia's PV structure for each IOP within this transformed coordinate framework. A bilinear interpolation was carried out for the transformation from $(r, z)$ space to $(R, \theta)$ space. In this coordinate space, the strong link between the diabatic heating field and the PV structure is apparent. The PV maxima are found just radially inward of the regions of heating, and evolve in concert with the changing structure and magnitude of the heating.

There is an approximately twofold increase in the maximum value of diabatic heating between RI and MI, followed by a corresponding decrease during RW. The increase in both magnitude and depth of the diabatic heating in the eyewall between RI and MI was accompanied by an approximate 100-PVU amplification of the hollow PV tower as Patricia just reached its maximum intensity. It is also noted that although the physical RMW contracted during this time period, in the $(R, \theta)$ space, the PV essentially concentrated "in place" (along the same $R$ surfaces) with the maximum remaining near $R=125 \mathrm{~km}$ (cf. Figs. 10a and 10b). The $M=0.8 \times 10^{6} \mathrm{~m}^{2} \mathrm{~s}^{-1}$ surface was shown earlier to approximately evolve with the RMW, and corresponds to the $R=188 \mathrm{~km}$ surface.

Equation (3) can be rearranged using Eq. (4) to yield the Eulerian PV equation in $(R, \theta)$ space, and with an application of the quotient rule, the terms can be combined to yield

$$
\frac{\partial P}{\partial \tau}=P^{2}\left[\frac{\partial}{R \partial R}\left(\frac{R \dot{R}}{P}\right)+\frac{\partial}{\partial \theta}\left(\frac{\dot{\theta}}{P}\right)\right] .
$$

The two terms on the right-hand side of Eq. (5) demonstrate that the evolution of PV at a fixed $(R, \theta)$ position depends on how the ratio of $\dot{R} / P$ changes radially along a 

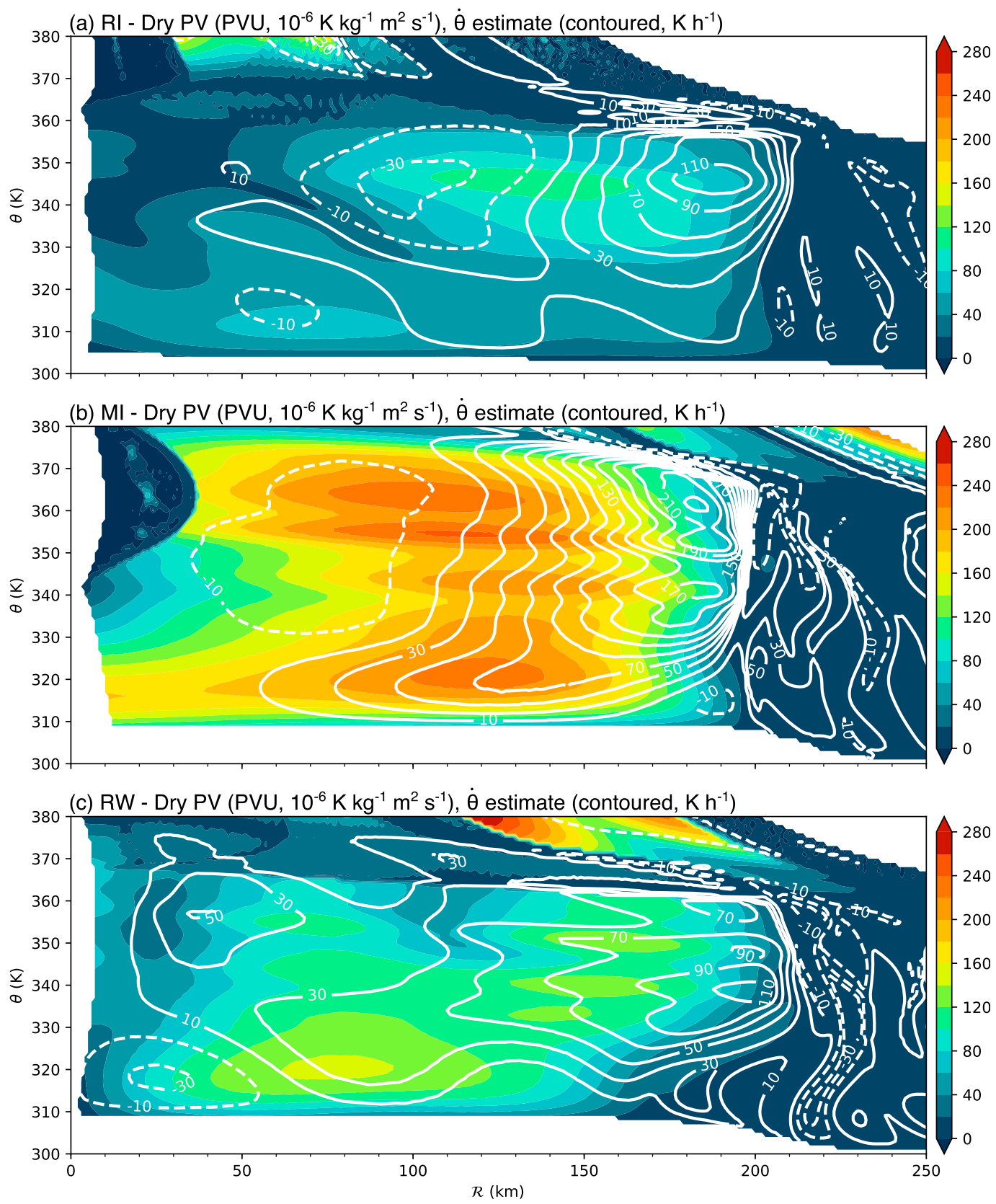

FIG. 10. Dry Ertel's PV (shading, PVU) and the diabatic heating $\dot{\theta}$ estimate (white, contours at $-20,50,100,150$, and $200 \mathrm{~K} \mathrm{~h}^{-1}$ ) are shown in potential radius and potential temperature $(R, \theta)$ space for the (a) RI, (b) MI, and (c) RW IOPs in Hurricane Patricia.

fixed $\theta$ surface, and how the ratio of $\dot{\theta} / P$ changes vertically along a fixed $R$ surface. The local tendency of PV is then scaled by the square of the preexisting $\mathrm{PV}$ at that location in $(R, \theta)$ space. A conceptual model depicting Patricia's rapid development of a hollow-tower PV structure can now be developed within this coordinate framework. As air parcels rise out of the boundary layer along an $R$ surface, a positive $P$ tendency requires the vertical gradient of $\dot{\theta} / P$ to be positive. Below the heating and $P$ maxima (which are vertically collocated; see Fig. 10a), a positive $P$ tendency occurs when the diabatic source of $P$ exceeds the vertical advection of smaller $P$ from below. Conversely, above the heating and $P$ maxima, a positive $P$ tendency occurs when the vertical advection of larger $P$ from below exceeds the diabatic sink of $P$. The local tendency resulting from these processes scales with $P^{2}$, supporting a rapid increase of $P$ in the presence of sustained vertical mass flux and heating. 
In the absence of eddy torques, a steady state is achieved when the vertical gradients of $\dot{\theta}$ and $P$ become identical, and the PV field becomes "locked" to the heating field (Hausman et al. 2006). As such, we would expect the PV maximum to be concentrated along the same $R$ surfaces and at the same isentropic level as the heating maximum, but the analyses indicate this is not the case. Instead, the heating maximum is located radially outward of the PV maximum, and remains nearly fixed to the same $R$ surfaces $(R \approx 180 \mathrm{~km})$ as they contract in physical space (cf. Figs. 5 and 7). This structural evolution during rapid intensification can be understood via two physical mechanisms. The axisymmetric generation of PV from diabatic processes results from projecting radial and vertical gradients of heating on to the vorticity vector. Since the vorticity vector in the eyewall region points radially outward and vertically upward, we would expect the generation of PV to occur both radially inward and beneath the diabatic heating maximum. However, the slope of the vorticity vector and heating distribution in the eyewall leads to a partial cancellation between these two individual terms. We can therefore infer that eddy processes must also be acting to concentrate the hollow PV tower radially inward of the heating maximum.

The first term on the right-hand side of Eq. (5) includes the effects of eddy torques [Eq. (2)] on the evolution of $\mathrm{PV}$ at a fixed $(R, \theta)$ position. Although we cannot calculate the eddy terms from the current analyses, we can draw inferences to their role in the observed development of Patricia's hollow-tower PV structure. Above the frictional boundary layer, we can reasonably assume a first-order local eddy viscosity turbulence closure that implies $\dot{R}$ would be maximized where the $M$ surfaces have the largest radial gradient in physical space, near the eye-eyewall interface (Figs. 4b, $6 \mathrm{~b}, 8 \mathrm{~b}$ ). Although eddy sources of momentum can also possess countergradient characteristics in TCs (Persing et al. 2013), we assume here that the dominant effect of eddy mixing is diffusive and downgradient. The concentration of the hollow PV tower near $R=125 \mathrm{~km}$ is consistent with Eq. (5) and the idea that diffusive eddy mixing provides a source of $P$ radially inward of the $\dot{R}$ maximum and a sink of $P$ radially outward of the maximum. The location of the $\dot{R}$ maximum moves radially inward in physical space as Patricia intensifies, but remains approximately fixed in potential radius space near $R=140 \mathrm{~km}$.

Collectively, the abovementioned heating and eddy processes can explain the observed in-place concentration of $\mathrm{PV}$ within the $(R, \theta)$ framework during $\mathrm{RI}$ and up through MI. Diabatic heating $\dot{\theta}$ concentrates PV near the same $R$ surfaces from RI to MI through vertical gradients of latent heating in the eyewall, with a positive feedback of local tendency as $P^{2}$ increases. Eddy torques $\dot{R}$ are maximized at the eye-eyewall interface radially inward of the heating maximum in the eyewall, which acts to concentrate PV near the eye-eyewall interface where the radial gradient of $\dot{R}$ is positive, and decrease it in the eyewall where the radial gradient of $\dot{R}$ is negative. During RW, the PV tower became diluted and expanded radially across $R$, suggesting that eddy mixing was occurring over a broader region as the eyewall broke down and asymmetries amplified. Despite the more complicated PV structure during RW, a strong correspondence with the diabatic heating field was still apparent (Fig. 10c).

As described in the introduction, the sign reversal of the radial PV gradient satisfies the Rayleigh necessary condition for barotropic instability, which may result in the development of mesovortices and enhanced PV mixing at the eye-eyewall interface. We argue here that this PV mixing process due to a barotropic or combined barotropic-baroclinic instability may have played an important role in the observed rapid weakening and vortex breakdown prior to landfall. To provide evidence supporting this argument, 3D Cartesian SAMURAI analyses were created for both the MI and RW periods. Utilizing the same data as the axisymmetric analyses (see Fig. 3), the Cartesian analyses were created on a grid with $1-\mathrm{km}$ horizontal spacing and $0.5-\mathrm{km}$ vertical spacing. Figure 11 shows the evolution of absolute vertical vorticity in Cartesian coordinates $(\hat{\eta}=f+\partial v / \partial x-\partial u / \partial y)$ between MI and RW at both 2- and 6-km altitude as a proxy for PV. The analysis captures an evolution consistent with rapid vorticity mixing as the vorticity "ring" apparent during MI developed into a structure more closely resembling a "monopole" during RW. Furthermore, through Stokes's theorem, the absolute circulation can be computed as the area integral of absolute vorticity within the domain bounded by each analysis shown in Fig. 11. In doing so, we find that the absolute circulation at each respective vertical level changes no more than $5 \%$ between MI and RW. The approximately conserved absolute circulation in the eye-eyewall region is consistent with the mechanism of vorticity mixing associated with barotropic instability (Schubert et al. 1999).

In the case of Patricia's evolution, the effects of vertical wind shear and formation of a secondary eyewall may have introduced potential pathways for barotropic instability to ensue. The effects of vertical wind shear likely played a role in reducing Patricia's vertical alignment, tilting the vortex between $\mathrm{MI}$ and $\mathrm{RW}$ and enhancing asymmetries (see Fig. 20 in Rogers et al. 2017). The disruption of the near-surface inflow to the primary eyewall by the developing secondary eyewall 
(a) $2 \mathrm{~km}(\mathrm{MI})$

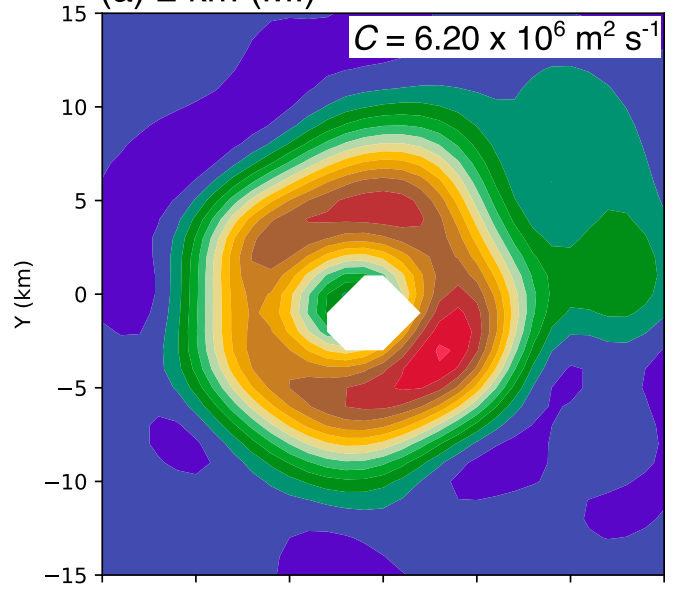

(c) $2 \mathrm{~km}(\mathrm{RW})$

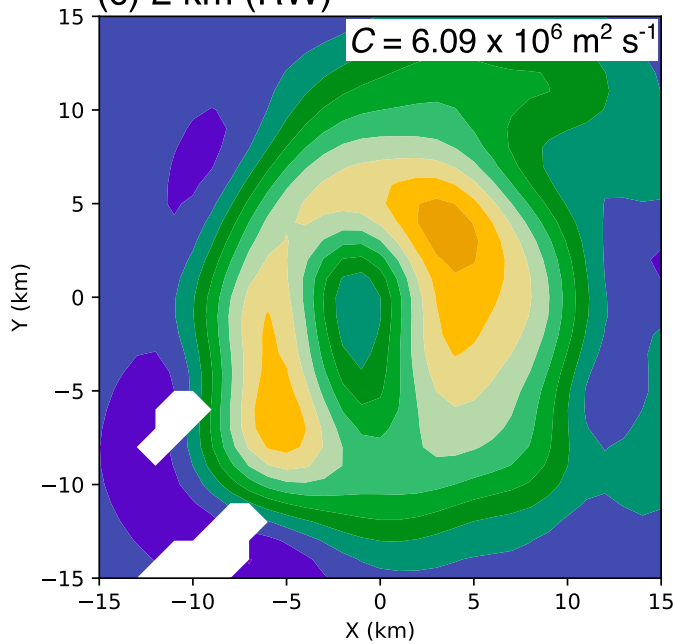

(b) $6 \mathrm{~km}(\mathrm{MI})$

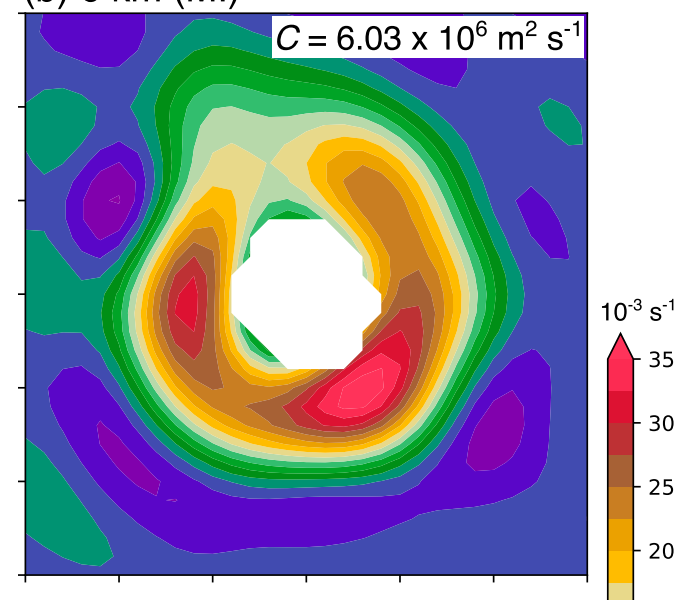

(d) $6 \mathrm{~km}(\mathrm{RW})$

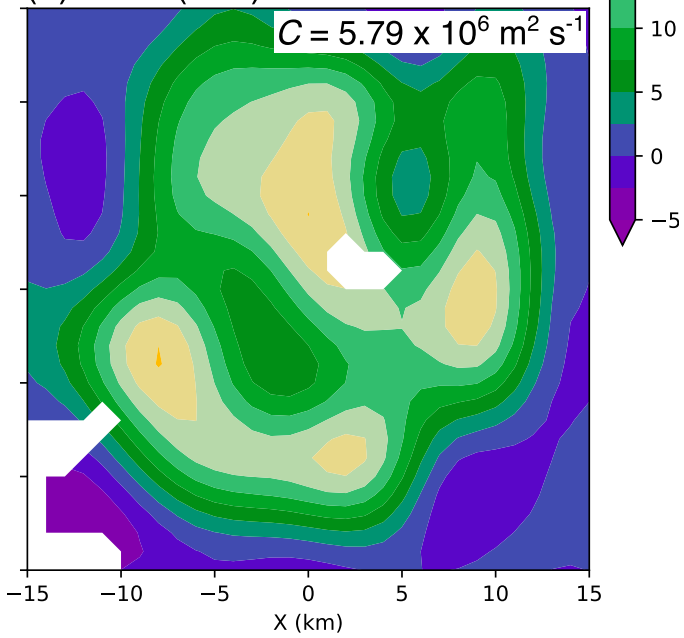

FIG. 11. Cartesian SAMURAI analysis for the MI and RW IOPs in Hurricane Patricia. Absolute vertical vorticity is shown at 2- and 6-km altitudes for (a),(b) MI and (c),(d) RW. The circulation computed within the horizontal domain bounded by these plots is shown in the upper-right corner of each panel.

was accompanied by a significant reduction in diabatic heating within the eyewall (cf. Figs. 7 and 9), which may have substantially reduced the forcing mechanism acting to concentrate PV along the inner edge of the eyewall. Another potential negative factor may have been the introduction of dry air from land being advected into Patricia as it approached the coast of Mexico. Although the present observational analysis precludes a detailed diagnosis of the relative importance of these different factors, the observed structural changes collectively suggest that multiple mechanisms led to Patricia's rapid weakening. It is difficult to explicitly calculate the most unstable modes and growth rates from the observations because of the strong sensitivity of the calculations to resolved gradients and spatial filtering. We hypothesize that a combination of internal and external factors sufficiently disrupted the diabatic forcing within
Patricia's primary eyewall, allowing instabilities to rapidly breakdown the symmetric PV ring and lead to enhanced mixing at the eye-eyewall interface. Further confirmation of this proposed mechanism for rapid weakening may require high-resolution numerical modeling.

\section{c. Comparisons to earlier observations}

Comparisons to the observed PV structure of other TCs are limited because of the difficulties in resolving the thermodynamic gradients required to calculate PV from observations. A midlevel PV maximum was noted in the western North Pacific composite analysis of Schubert and Hack (1983, their Fig. 4) and in Hurricane Gloria of 1985 (Shapiro and Franklin 1995, their Fig. 11). Bell and Montgomery (2008) highlighted a low-level PV maximum in the eye of Hurricane Isabel (2003), which is 


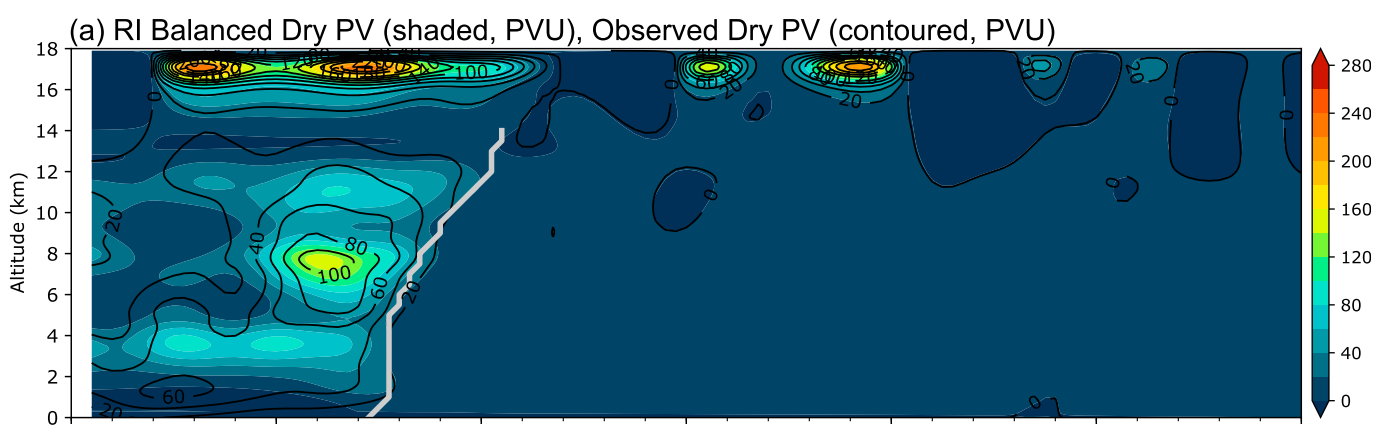

(b) RW Balanced Dry PV (shaded, PVU), Observed Dry PV (contoured, PVU)

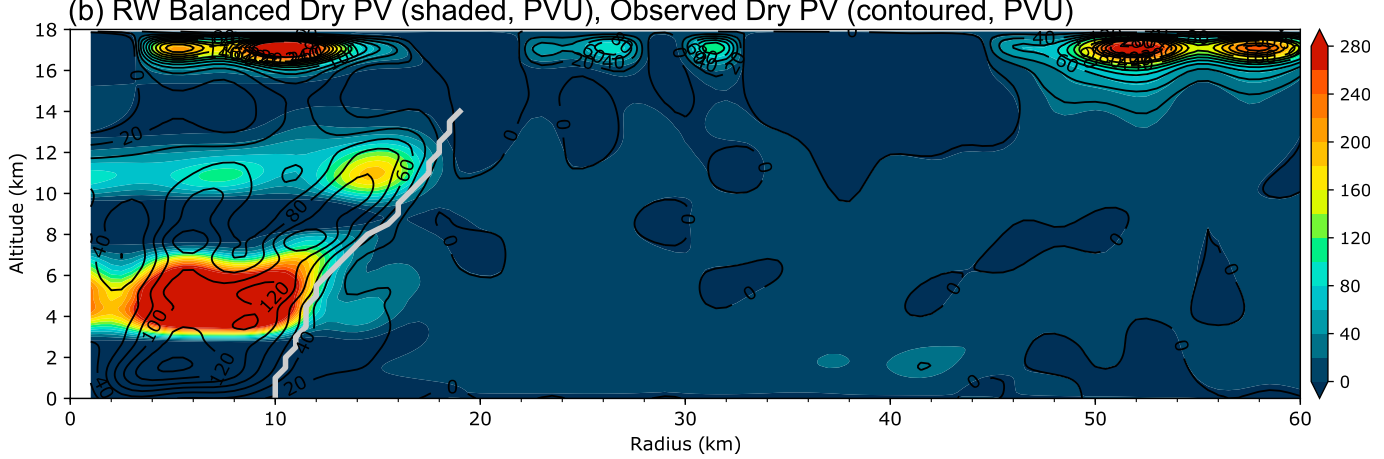

FIG. 12. "Balanced" potential vorticity comparison for the (a) RI and (b) RW IOPs. PV calculated from the balance assumption (shaded, PVU) is overlaid with PV computed from each respective SAMURAI analysis (contoured, PVU) using the same 20-PVU contour interval. See section 5c for details.

similar to that seen in Hurricane Patricia. This "PV bridge" has been attributed to preferential PV mixing at low levels where both the shear and curvature vorticity are maximized, leading to a rapid growth of the most unstable modes (Hendricks and Schubert 2010). Hendricks and Schubert (2010) also attributed this local PV maximum to the enhanced stratification found near the subsidence inversion in the eye. Recent studies have shown that high PV mixed from the eyewall into the eye at low levels can contribute to increased stratification, suggesting that the low-level inversion itself may in part be dynamically driven through PV mixing (Rozoff et al. 2009; Hendricks and Schubert 2010).

As Patricia reached its maximum intensity, the magnitude of PV concentrated within the hollow tower was approximately 3-4 times greater than that observed in Gloria (Shapiro and Franklin 1995; Shapiro 1996) and Isabel (Bell and Montgomery 2008). A similar structure was documented by Hausman et al. (2006), who utilized an axisymmetric model to simulate a TC that developed a hollow tower of PV with a maximum of $\sim 275$ PVU. The compact RMW, deep tower of PV with a maximum near midlevels, and intense wind speeds of the simulated TC in Hausman et al. are remarkably similar to Patricia, and suggest that axisymmetric mechanisms can provide a reasonable first-order approximation to explain Patricia's rapid intensification. However, the idealized
TC in Hausman et al. (2006) does not weaken after achieving maximum intensity. As was hypothesized above, a combination of internal asymmetric processes and external environmental influences is necessary to explain Patricia's observed rapid weakening.

Earlier studies that attempted to calculate PV in a TC had to rely on determining the mass field via a balance assumption, whereby the observed winds are used to calculate the mass field given an imposed balance condition (e.g., Schubert and Hack 1983; Shapiro and Franklin 1995; Shapiro 1996). To examine the validity of a balance assumption, we calculated PV using the mass fields that would be in gradient wind and hydrostatic balance with the observed wind fields for both the RI and RW analyses ${ }^{3}$ following the method described by Smith (2006) and Foerster and Bell (2017). During RI, PV calculated using the balanced mass field is qualitatively similar to the analyzed PV maximum in the midtroposphere, but shows distinct quantitative differences, especially in the lower troposphere and outflow layer where the "balanced" PV is much lower (Fig. 12a). Differences are largest in the regions where gradient

\footnotetext{
${ }^{3}$ Since the MI analysis utilized the thermodynamic analysis valid during RW, a comparison to the mass field computed using a balance assumption during MI would be indirect.
} 
wind balance is a poor approximation for the observed winds. The balanced mass field is particularly problematic for calculating PV during the RW period (Fig. 12b) given that the tangential velocity increases with height up to its maximum near 5-km altitude (Fig. 8), requiring that the isentropes slope upward approaching the axis of rotation via thermal wind balance (i.e., a cold-core cyclone). Compounding errors result when computing the gradients of unrealistic isentropic structures (not shown), which is required to calculate PV. Similar structural issues resulting from using a balance assumption were noted by Shapiro and Franklin (1995). We conclude that the balance assumption is a reasonable first-order approximation above the boundary layer and below the outflow layer during the RI analysis, but is largely invalid during the RW analysis because of the presence of large regions with unbalanced flow. These results suggest that the high-resolution thermodynamic observations obtained from the XDDs are crucial in permitting the calculations of PV presented herein.

\section{Conclusions}

A new observational dataset created from the Tropical Cyclone Intensity experiment (TCI; Doyle et al. 2017) and the Intensity Forecasting Experiment (IFEX; Rogers et al. 2006, 2013a) field campaigns provided an opportunity to investigate the record-breaking evolution of Hurricane Patricia (2015). Dropsondes released with high spatial resolution from approximately $18-\mathrm{km}$ altitude allowed for an unprecedented full-tropospheric analysis of a TC experiencing rapid intensity change. The analyses presented herein highlight the first fulltropospheric, axisymmetric calculations of Ertel's potential vorticity (PV) within the inner core of a TC, derived from observations without the use of balance assumptions. Observations gathered during three intensive observing periods (IOPs) by the NOAA P-3 and NASA WB-57 aircraft provided information at three critical stages during Patricia's life cycle: the rapid intensification phase (RI), near maximum intensity (MI), and the rapid weakening phase (RW).

During the rapid intensification phase, Patricia's structure was characterized by a hollow-tower PV structure concentrated along the inner edge of the RMW. The lowlevel eye (beneath $\sim 3-\mathrm{km}$ altitude) exhibited a secondary PV maximum that was related to both the subsidence inversion and mixing at the eye-eyewall interface. The present analysis confirms the coexistence of a PV bridge at low levels and a hollow-tower PV structure with a midlevel maximum during rapid intensification. Near maximum intensity, the hollow PV tower extended throughout the depth of the troposphere with peak values exceeding 250 PVU, making this the highest tropospheric PV documented from observations to the authors' knowledge. Discrete maxima of PV were also found in the uppertroposphere-lower-stratosphere (UTLS) region associated with strong static stability. These upper-level PV rings have been noted in past numerical simulations (e.g., Kieu and Zhang 2010), but their role in Patricia's intensity change is currently unclear and warrants further investigation.

The hollow-tower PV structure observed in Patricia's eyewall supported the Charney-Stern necessary condition for combined barotropic-baroclinic instability. Patricia's rapid weakening over a period of $3 \mathrm{~h}$ was influenced by increasing vertical wind shear, a secondary eyewall, and possibly by dry air from the nearby landmass, which combined to reduce the symmetric heating in the eyewall. Evidence of vorticity mixing at the eyeeyewall interface and approximate conservation of absolute circulation in this region suggest that barotropic or combined barotropic-baroclinic instability may have played an important role in the rapid weakening. The combined effects of these internal and external processes resulted in a broader, weaker PV tower with maximum values of $\sim 160$ PVU.

Additional insight to explain Patricia's evolution was provided by transforming the PV analyses to potential radius and potential temperature $(R, \theta)$ coordinates. The rapid intensification of Patricia's hollow PV tower within this framework essentially occurred "in place" in a region of approximately constant potential radius, even though the eyewall contracted in physical space. The Eulerian PV tendency equation in $(R, \theta)$ coordinates indicates that $\mathrm{PV}$ is concentrated more quickly in regions with preexisting large values of $\mathrm{PV}$, suggesting that the rapid vertical development of the PV tower resulted from sustained vertical mass flux and heating in this region. The location of the PV maximum near the eye-eyewall interface, radially inward of the heating maximum, can be further explained by the effects of eddy mixing along the interface. The rapid breakdown of Patricia's hollow PV tower during the transition from MI to RW can be explained within this framework as resulting from increased eddy mixing over a broader region, but a strong correspondence of the PV field with the symmetric heating distribution is still evident during this period. The absolute circulation was shown to be approximately conserved during the rapid weakening, substantiating the mechanism for eddy-driven vortex breakdown put forth in earlier idealized work (e.g., Schubert et al. 1999).

The observational analyses presented herein illustrate the evolution of Hurricane Patricia within an axisymmetric framework, and provide some validation of the PV conceptual model to provide insight into rapid TC intensity changes. However, the present axisymmetric 
PV analyses are fundamentally limited in their representation of Patricia's full 3D evolution, both temporally and spatially. While the calculation of 3D Ertel's PV from observations is difficult, it may be possible with a combination of high-resolution dropsondes and Doppler radar data in future observational analyses of TCs, which can help provide further insight into TC evolution. Along with these observations, high-resolution numerical simulations are necessary to examine the key processes contributing to rapid intensity changes and to better predict TC intensity prior to landfall. Future work will further investigate the role of asymmetric processes during Patricia's evolution, and compare the observed features presented herein with other TCs to further explore the utility of the PV framework for understanding TC structure and intensity change.

Acknowledgments. This work was funded by the Office of Naval Research awards N000141613033 and N000141410118 under the Tropical Cyclone Intensity Departmental Research Initiative. MMB was also supported by the National Science Foundation (NSF) Award AGS-1701225, and JM was supported by the NSF Bridge to the Doctorate Fellowship Award 004863-00003. We thank the crews of both the NOAA P-3 and the NASA WB-57, and the TCI Science Team for collecting and quality controlling the observations used in this study. Insightful discussions with Wayne $\mathrm{H}$. Schubert aided in developing the potential vorticity conceptual model presented in this study. We also thank Dave Nolan and one anonymous reviewer for helpful comments, which improved the overall quality of the manuscript.

\section{REFERENCES}

Banzon, V., T. M. Smith, T. M. Chin, C. Liu, and W. Hankins, 2016: A long-term record of blended satellite and in situ sea-surface temperature for climate monitoring, modeling and environmental studies. Earth Syst. Sci. Data, 8, 165-176, https:// doi.org/10.5194/essd-8-165-2016.

Bell, M. M., and M. T. Montgomery, 2008: Observed structure, evolution, and potential intensity of category 5 Hurricane Isabel (2003) from 12 to 14 September. Mon. Wea. Rev., 136, 2023-2046, https://doi.org/10.1175/2007MWR1858.1.

_, _ , and K. A. Emanuel, 2012: Air-sea enthalpy and momentum exchange at major hurricane wind speeds observed during CBLAST. J. Atmos. Sci., 69, 3197-3122, https://doi.org/ 10.1175/JAS-D-11-0276.1.

— W.-C. Lee, C. A. Wolff, and H. Cai, 2013: A solo-based automated quality control algorithm for airborne tail Doppler radar data. J. Appl. Meteor. Climatol., 52, 2509-2528, https:// doi.org/10.1175/JAMC-D-12-0283.1.

, and Coauthors, 2016: ONR tropical cyclone intensity 2015 NASA WB-57 dropsonde data, version 1.0. UCAR-NCAR Earth Observing Laboratory, accessed 23 January 2017, https:// doi.org/10.5065/D6KW5D8M.
Black, P., L. Harrison, M. Beaubien, R. Bluth, R. Woods, A. Penny, R. W. Smith, and J. D. Doyle, 2017: High-Definition Sounding System (HDSS) for atmospheric profiling. J. Atmos. Oceanic Technol., 34, 777-796, https://doi.org/10.1175/JTECH-D-14-00210.1.

Cai, H., W.-C. Lee, M. M. Bell, C. A. Wolff, X. Tang, and F. Roux, 2018: A generalized navigation correction method for airborne Doppler radar data. J. Atmos. Oceanic Technol., 35, 1999-2017, https://doi.org/10.1175/JTECH-D-18-0028.1.

DeMaria, M., M. Mainelli, L. K. Shay, J. A. Knaff, and J. Kaplan, 2005: Further improvements to the Statistical Hurricane Intensity Prediction Scheme (SHIPS). Wea. Forecasting, 20, 531-543, https://doi.org/10.1175/WAF862.1.

—, C. R. Sampson, J. A. Knaff, and K. D. Musgrave, 2014: Is tropical cyclone intensity guidance improving? Bull. Amer. Meteor. Soc., 95, 387-398, https://doi.org/10.1175/BAMS-D12-00240.1.

Doyle, J. D., and Coauthors, 2017: A view of tropical cyclones from above: The Tropical Cyclone Intensity (TCI) experiment. Bull. Amer. Meteor. Soc., 98, 2113-2134, https://doi.org/ 10.1175/BAMS-D-16-0055.1.

Eastin, M. D., P. G. Black, and W. M. Gray, 2002: Flight-level thermodynamic instrument wetting errors in hurricanes. Part I: Observations. Mon. Wea. Rev., 130, 825-841, https://doi.org/ 10.1175/1520-0493(2002)130<0825:FLTIWE > 2.0.CO;2.

Eliassen, E., 1951: Slow thermally or frictionally controlled meridional circulation in a circular vortex. Astrophys. Nor., 5, 19-60.

Ertel, H., 1942: Ein neuer hydrodynamischer erhaltungssatz. Naturwissenschaften, 30, 543-544, https://doi.org/10.1007/BF01475602.

Foerster, A. M., and M. M. Bell, 2017: Thermodynamic retrieval in rapidly rotating vortices from multiple-Doppler radar data. J. Atmos. Oceanic Technol., 34, 2353-2374, https://doi.org/ 10.1175/JTECH-D-17-0073.1.

,-- P. A. Harr, and S. C. Jones, 2014: Observations of the eyewall structure of Typhoon Sinlaku (2008) during the transformation stage of extratropical transition. Mon. Wea. Rev., 142, 3372-3392, https://doi.org/10.1175/MWR-D-13-00313.1.

Franklin, J. L., S. J. Lord, and F. D. Marks Jr., 1988: Dropwindsonde and radar observations of the eye of Hurricane Gloria (1985). Mon. Wea. Rev., 116, 1237-1244, https://doi.org/ 10.1175/1520-0493(1988)116<1237:DAROOT>2.0.CO;2.

Guinn, T. A., and W. H. Schubert, 1993: Hurricane spiral bands. J. Atmos. Sci., 50, 3380-3403, https://doi.org/10.1175/15200469(1993) $050<3380: \mathrm{HSB}>2.0 . \mathrm{CO} ; 2$.

Hack, J. J., and W. H. Schubert, 1986: Nonlinear response of atmospheric vortices to heating by organized cumulus convection. J. Atmos. Sci., 43, 1559-1573, https://doi.org/10.1175/ 1520-0469(1986)043<1559:NROAVT>2.0.CO;2.

Hausman, S. A., V. K. Ooyama, and W. H. Schubert, 2006: Potential vorticity structure of simulated hurricanes. J. Atmos. Sci., 63, 87-108, https://doi.org/10.1175/JAS3601.1.

Haynes, P. H., and M. E. McIntyre, 1987: On the evolution of vorticity and potential vorticity in the presence of diabatic heating and frictional or other forces. J. Atmos. Sci., 44, 828-841, https:// doi.org/10.1175/1520-0469(1987)044<0828:OTEOVA>2.0.CO;2.

Hendricks, E. A., and W. H. Schubert, 2010: Adiabatic rearrangement of hollow PV towers. J. Adv. Model. Earth Syst., 2 (1), https://doi.org/10.3894/JAMES.2010.2.2.

,-- R. K. Taft, H. Wang, and J. P. Kossin, 2009: Life cycles of hurricane-like vorticity rings. J. Atmos. Sci., 66, 705-722, https://doi.org/10.1175/2008JAS2820.1.

, M. S. Peng, B. Fu, and T. Li, 2010: Quantifying environmental control on tropical cyclone intensity change. Mon. Wea. Rev., 138, 3243-3271, https://doi.org/10.1175/2010MWR3185.1. 
- B. D. McNoldy, and W. H. Schubert, 2012: Observed innercore structural variability in Hurricane Dolly (2008). Mon. Wea. Rev., 140, 4066-4077, https://doi.org/10.1175/MWR-D12-00018.1.

, W. H. Schubert, Y.-H. Chen, H.-C. Kuo, and M. S. Peng, 2014: Hurricane eyewall evolution in a forced shallow-water model. J. Atmos. Sci., 71, 1623-1643, https://doi.org/10.1175/ JAS-D-13-0303.1.

Hoskins, B. J., M. E. McIntyre, and A. W. Robertson, 1985: On the use and significance of isentropic potential vorticity maps. Quart. J. Roy. Meteor. Soc., 111, 877-946, https://doi.org/ 10.1002/qj.49711147002.

Houze, R. A., Jr., S. S. Chen, B. F. Smull, W.-C. Lee, and M. M. Bell, 2007: Hurricane intensity and eyewall replacement. $\mathrm{Na}$ ture, 315, 1235-1239, https://doi.org/10.1126/science.1135650.

Jordan, C. L., 1961: Marked changes in the characteristics of the eye of intense typhoons between the deepening and filling stages. J. Meteor., 18, 779-789, https://doi.org/10.1175/15200469(1961)018<0779:MCITCO > 2.0.CO;2.

Kaplan, J., M. DeMaria, and J. A. Knaff, 2010: A revised tropical cyclone rapid intensification index for the Atlantic and eastern North Pacific basins. Wea. Forecasting, 25, 220-241, https:// doi.org/10.1175/2009WAF2222280.1.

—_, and Coauthors, 2015: Evaluating environmental impacts on tropical cyclone rapid intensification predictability utilizing statistical models. Wea. Forecasting, 30, 1374-1396, https:// doi.org/10.1175/WAF-D-15-0032.1.

Kieu, C. Q., and D.-L. Zhang, 2010: A piecewise potential vorticity inversion algorithm and its application to hurricane inner-core anomalies. J. Atmos. Sci., 67, 2616-2131, https://doi.org/ 10.1175/2010JAS3421.1.

Kimberlain, T. B., E. S. Blake, and J. P. Cangialosi, 2016: Hurricane Patricia. National Hurricane Center Tropical Cyclone Rep., $32 \mathrm{pp}$.

Kossin, J. P., and M. D. Eastin, 2001: Two distinct regimes in the kinematic and thermodynamic structure of the hurricane eye and eyewall. J. Atmos. Sci., 58, 1079-1090, https://doi.org/ 10.1175/1520-0469(2001)058<1079:TDRITK > 2.0.CO;2.

— patterns, and rapid pressure falls in hurricane-like vortices. J. Atmos. Sci., 58, 2196-2209, https://doi.org/10.1175/15200469(2001)058<2196:MPFPAR > 2.0.CO;2.

Marks, F. D., R. A. Houze Jr., and J. Gamache, 1992: Dual-aircraft investigation of the inner core of Hurricane Norbert. Part I: Kinematic structure. J. Atmos. Sci., 49, 919-942, https://doi.org/ 10.1175/1520-0469(1992)049<0919:DAIOTI>2.0.CO;2.

Martinez, J., M. M. Bell, J. L. Vigh, and R. F. Rogers, 2017: Examining tropical cyclone structure and intensification with the FLIGHT+ dataset from 1999 to 2012. Mon. Wea. Rev., 145, 4401-4421, https://doi.org/10.1175/MWR-D-17-0011.1.

Möller, J. D., and R. K. Smith, 1994: The development of potential vorticity in a hurricane-like vortex. Quart. J. Roy. Meteor. Soc., 120, 1255-1265, https://doi.org/10.1002/qj.49712051907.

—_, and M. T. Montgomery, 1999: Vortex Rossby waves and hurricane intensification in a barotropic model. J. Atmos. Sci., 56, 1674-1687, https://doi.org/10.1175/1520-0469(1999)056<1674: VRWAHI $>2.0 . \mathrm{CO} ; 2$.

$\longrightarrow$, and — 2000: Tropical cyclone evolution via potential vorticity anomalies in a three-dimensional balance model. J. Atmos. Sci., 57, 3366-3387, https://doi.org/10.1175/15200469(2000)057<3366:TCEVPV>2.0.CO;2.

Montgomery, M. T., and L. J. Shapiro, 1995: Generalized CharneyStern and Fjortoft theorems for rapidly rotating vortices.
J. Atmos. Sci., 52, 1829-1833, https://doi.org/10.1175/15200469(1995)052<1829:GCAFTF>2.0.CO;2.

_ , and R. J. Kallenbach, 1997: A theory for vortex Rossbywaves and its application to spiral bands and intensity changes in hurricanes. Quart. J. Roy. Meteor. Soc., 123, 435-465, https://doi.org/10.1002/qj.49712353810.

Ooyama, K. V., 2002: The cubic-spline transform method: Basic definitions and tests in a 1D single domain. Mon. Wea. Rev. 130, 2392-2415, https://doi.org/10.1175/1520-0493(2002) $130<2392:$ TCSTMB $>2.0$. CO 2 .

Pendergrass, A. G., and H. E. Willoughby, 2009: Diabatically induced secondary flows in tropical cyclones. Part I: Quasisteady forcing. Mon. Wea. Rev., 137, 805-821, https://doi.org/ 10.1175/2008MWR2657.1.

Persing, J., M. T. Montgomery, J. C. McWilliams, and R. K. Smith, 2013: Asymmetric and axisymmetric dynamics of tropical cyclone intensification. Atmos. Chem. Phys., 13, 12 299-12 341, https://doi.org/10.5194/acp-13-12299-2013.

Purser, R. J., W.-S. Wu, D. F. Parrish, and N. M. Roberts, 2003: Numerical aspects of the application of recursive filters to variational statistical analysis. Part I: Spatially homogeneous and isotropic Gaussian covariances. Mon. Wea. Rev., 131, 1524 1535, https://doi.org/10.1175//1520-0493(2003)131<1524: NAOTAO $>2.0 . \mathrm{CO} ; 2$.

Rogers, R., and Coauthors, 2006: The Intensity Forecasting Experiment (IFEX): A NOAA multiyear field program for improving tropical cyclone intensity forecasts. Bull. Amer. Meteor. Soc., 87, 1523-1537, https://doi.org/10.1175/BAMS-87-11-1523.

_ _ P. Reasor, and S. Lorsolo, 2013a: Airborne Doppler observations of the inner-core structural differences between intensifying and steady-state tropical cyclones. Mon. Wea. Rev., 141, 2970-2991, https://doi.org/10.1175/MWR-D-12-00357.1.

- and Coauthors, 2013b: NOAA's Hurricane Intensity Forecasting Experiment: A progress report. Bull. Amer. Meteor. Soc., 94, 859-882, https://doi.org/10.1175/BAMS-D-12-00089.1.

_ P. D. Reasor, and J. A. Zhang, 2015: Multiscale structure and evolution of Hurricane Earl (2010) during rapid intensification. Mon. Wea. Rev., 143, 536-562, https://doi.org/10.1175/ MWR-D-14-00175.1.

- and Coauthors, 2017: Rewriting the tropical record books: The extraordinary intensification of Hurricane Patricia (2015). Bull. Amer. Meteor. Soc., 98, 2091-2112, https://doi.org/ 10.1175/BAMS-D-16-0039.1.

Roux, F., V. Marécal, and D. Hauser, 1993: The 12/13 January 1988 narrow cold-frontal rainband observed during MFDP/ FRONTS 87. Part I: Kinematics and thermodynamics. J. Atmos. Sci., 50, 951-974, https://doi.org/10.1175/15200469(1993)050<0951:TJNCFR $>2.0$.CO;2.

Rozoff, C. M., J. P. Kossin, W. H. Schubert, and P. J. Mulero, 2009: Internal control of hurricane intensity variability: The dual nature of potential vorticity mixing. J. Atmos. Sci., 66, 133147, https://doi.org/10.1175/2008JAS2717.1.

_ C. S. Velden, J. Kaplan, J. P. Kossin, and A. J. Wimmers, 2015: Improvements in the probabilistic prediction of tropical cyclone rapid intensification with passive microwave observations. Wea. Forecasting, 30, 1016-1038, https://doi.org/ 10.1175/WAF-D-14-00109.1.

Schubert, W. H., 2004: A generalization of Ertel's potential vorticity to a cloudy, precipitating atmosphere. Meteor. Z., 13, 465-471, https://doi.org/10.1127/0941-2948/2004/0013-0465.

_ , and J. J. Hack, 1982: Inertial stability and tropical cyclone development. J. Atmos. Sci., 39, 1687-1697, https://doi.org/ 10.1175/1520-0469(1982)039<1687:ISATCD>2.0.CO;2. 
— model. J. Atmos. Sci., 40, 1571-1583, https://doi.org/10.1175/ 1520-0469(1983)040<1571:TEBVM >2.0.CO;2.

— , and B. T. Alworth, 1987: Evolution of potential vorticity in tropical cyclones. Quart. J. Roy. Meteor. Soc., 113, 147-162, https://doi.org/10.1002/qj.49711347509.

— M. T. Montgomery, R. K. Taft, T. A. Guinn, S. R. Fulton, J. Kossin, and J. P. Edwards, 1999: Polygonal eyewalls, asymmetric eye contraction, and potential vorticity mixing in hurricanes. J. Atmos. Sci., 56, 1197-1223, https://doi.org/ 10.1175/1520-0469(1999)056<1197:PEAECA > 2.0.CO;2.

— S. A. Hausman, M. Garcia, K. V. Ooyama, and H.-C. Kuo, 2001: Potential vorticity in a moist atmosphere. J. Atmos. Sci., 58, 3148-3157, https://doi.org/10.1175/1520-0469(2001)058<3148: PVIAMA $>2.0 . \mathrm{CO} ; 2$.

Shapiro, L. J., 1996: The motion of Hurricane Gloria: A potential vorticity diagnosis. Mon. Wea. Rev., 124, 2497-2508, https://doi.org/ 10.1175/1520-0493(1996)124<2497:TMOHGA > 2.0.CO;2.

— , and H. E. Willoughby, 1982: The response of balanced hurricanes to local sources of heat and momentum. J. Atmos. Sci., 39, 378-394, https://doi.org/10.1175/1520-0469(1982)039<0378: TROBHT $>2.0 . \mathrm{CO} ; 2$.

— , and J. L. Franklin, 1995: Potential vorticity in Hurricane Gloria. Mon. Wea. Rev., 123, 1465-1475, https://doi.org/ 10.1175/1520-0493(1995)123<1465:PVIHG > 2.0.CO;2.

Smith, R. K., 2006: Accurate determination of a balanced axisymmetric vortex in a compressible atmosphere. Tellus, $\mathbf{5 8 A}$, 98-103, https://doi.org/10.1111/j.1600-0870.2006.00149.x.

— and M. T. Montgomery, 2016: The efficiency of diabatic heating and tropical cyclone intensification. Quart. J. Roy. Meteor. Soc., 142, 2081-2086, https://doi.org/10.1002/qj.2804.

, _ - and H. Bui, 2018: Axisymmetric balance dynamics of tropical cyclone intensification and its breakdown revisited. J. Atmos. Sci., 75, 3169-3189, https://doi.org/10.1175/JAS-D-17-0179.1.
Stern, D. P., and D. S. Nolan, 2009: Reexamining the vertical structure of tangential winds in tropical cyclones: Observations and theory. J. Atmos. Sci., 66, 3579-3600, https://doi.org/ 10.1175/2009JAS2916.1.

, and F. Zhang, 2013: How does the eye warm? Part I: A potential temperature budget analysis of an idealized tropical cyclone. J. Atmos. Sci., 70, 73-90, https://doi.org/10.1175/JASD-11-0329.1.

_ J. J. R. Brisbois, and D. S. Nolan, 2014: An expanded dataset of hurricane eyewall sizes and slopes. J. Atmos. Sci., 71, 27472762, https://doi.org/10.1175/JAS-D-13-0302.1.

Velden, C. S., C. M. Hayden, S. Nieman, W. P. Menzel, and J. Goerss, 1997: Upper-tropospheric winds derived from geostationary satellite water vapor observations. Bull. Amer. Meteor. Soc., 78, 173-195, https://doi.org/10.1175/ 1520-0477(1997)078<0173:UTWDFG > 2.0.CO;2.

- , and Coauthors, 2005: Recent innovations in deriving tropospheric winds from meteorological satellites. Bull. Amer. Meteor. Soc., 86, 205-223, https://doi.org/10.1175/BAMS-862-205.

Vigh, J. L., and W. H. Schubert, 2009: Rapid development of the tropical cyclone warm core. J. Atmos. Sci., 66, 3335-3350, https://doi.org/10.1175/2009JAS3092.1.

Willoughby, H. E., 1998: Tropical cyclone eye thermodynamics. Mon. Wea. Rev., 126, 3053-3067, https://doi.org/10.1175/15200493(1998)126<3053:TCET>2.0.CO;2.

Zhang, D.-L., Y. Liu, and M. K. Yau, 2002: A multiscale numerical study of Hurricane Andrew (1992). Part V: Inner-core thermodynamics. Mon. Wea. Rev., 130, 2745-2763, https://doi.org/ 10.1175/1520-0493(2002)130<2745:AMNSOH>2.0.CO;2.

Zipser, E. J., R. J. Meitin, and M. A. LeMone, 1981: Mesoscale motion fields associated with slowly moving gate convective band. J. Atmos. Sci., 38, 1725-1750, https://doi.org/10.1175/ 1520-0469(1981)038<1725:MMFAWA > 2.0.CO;2. 